\title{
A free boundary problem in glaciology: The motion of grounding lines
}

\author{
MARCO A. FONTELOS ${ }^{\dagger}$ \\ Departamento de Matemáticas, Universidad Autónoma de Madrid, 28049 Madrid, Spain \\ AND \\ ANA I. MUÑOZ \\ Departamento de Matemáticas, Física Aplicada y Ciencias de la Naturaleza, \\ Universidad Rey Juan Carlos, 28933, Móstoles, Madrid, Spain
}

[Received 12 January 2006 and in revised form 14 July 2006]

\begin{abstract}
We consider stationary ice sheet modeled as a Stokes flow in a bounded two-dimensional domain. In particular, we study the behavior of the grounding line, where different boundary conditions meet: no-slip conditions for the grounded part and force balance conditions for the floating part whose shape is a priori undetermined. This yields a free boundary problem with mixed boundary conditions and a contact line, called "grounding line" in the glaciological context, that might move along the solid substrate. We show that solutions with moving grounding lines and zero contact angle do exist and determine the shape and asymptotic properties of the free boundary.
\end{abstract}

Keywords: Free boundary problem; glaciology; mixed type boundary conditions; Stokes' flow problem; Lax-Milgram theorem; Mellin transform.

\section{Introduction}

In this paper we study a free boundary problem arising in a glaciological context and involving the motion of a contact line separating a solid from a fluid interface. The contact line is called, in this context, "grounding line" and the question on whether or not it can move and which are the dominant mechanisms is subject of debate. Ice sheets are typically separated into three distinct flow regimes, which are referred to as inland ice sheet, ice streams (or outlet glaciers of fast flow features) and ice shelves (see Figure 1). The interaction of these three components is crucial in determining the evolution of the whole ice-sheet system. Ice shelves may form if local ice thickness is insufficient to prevent floatation in the surrounding oceans. The grounding line separates the grounded ice zone from the floating zone, and each zone has its distinct flow regime. Some distance away from this grounding zone, either in the ice shelf or in the ice sheet, ice flow is reasonably well understood and there is some consensus as to which simplifications can be made in the stress equilibrium, either to have flow dominated by shearing in horizontal planes, most of it at the base as in the ice sheet, or to have the flow dominated by lateral shearing and longitudinal stretching, as in the ice shelf. This fundamental difference between the two flow regimes seems to suggest the existence of a transition zone where all the stress components are important and no simplification can be made. There also

\footnotetext{
†E-mail: marco.fontelos@uam.es

‡E-mail: anaisabel.munoz@urjc.es
} 


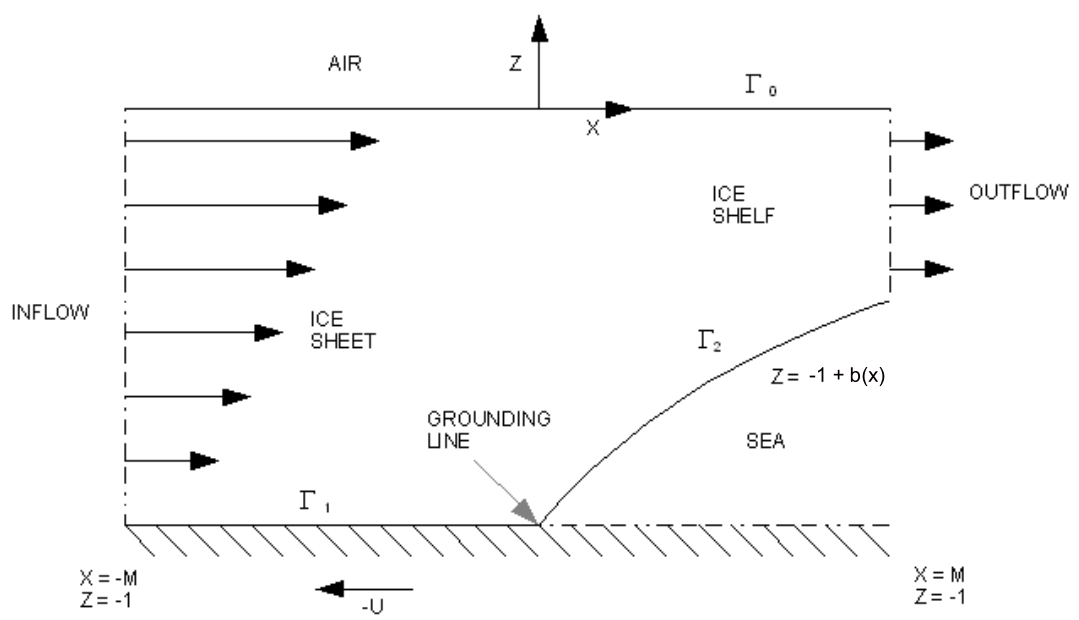

FIG. 1. Physical setting of the problem.

arises the question on the width of such a transition zone, if any, which is moreover likely to be strongly influenced by the occurrence of basal sliding or the development of ice streams.

On the other hand, it has also been suggested that the details of the stress and strain conditions at the grounding line would be unimportant for modeling grounding-line migration, as the inland flow would be little influenced by conditions in the ice shelf, and grounding-line migration could be above all controlled by conditions of hydrostatic equilibrium in the grounding area. Whatever the case, grounding-line migration and the coupling of ice sheet flow with ice shelf flow can rightly be considered as one of those "grand unsolved modeling problems", which is nevertheless of prime importance because it is the predominant mechanism by which Antarctic ice sheet changes its dimensions (it is a key process affecting the stability of marine ice sheets such as the West Antarctic Ice Sheet (WAIS)). Only a few studies have tried to deal with the numerical inconsistencies at the grounding line that most finite difference models suffer from (see [12]). In these models the focus is on the dynamical problem and less attention is paid to the mechanical coupling between the ice sheet and ice shelf. In the works [28, 29, 6, 6] some models of marine ice stream flow and in particular two-dimensional steady isothermal flow are developed.

The model studied in this paper is based on the models developed in the above mentioned references. In [28, 29] a consistent scaling is applied to consider ice streams with different relations between the shear and longitudinal deviatoric stresses and a parametric analysis is performed in order to prescribe suitable governing equations at the ice stream-ice shelf transition zone depending on different physical assumptions. Wilchinsky and Chugunov conclude that if the shear stress in the ice stream is much larger than the longitudinal stress deviator, then all the stresses in the transition zone are of the same order of magnitude and the transition zone should be singular. However, if the importance of the shearing component is small near the grounding line and one assumes that the solution for the ice shelf is valid up to the grounding line, then the problem of marine-ice stream dynamics determines the ice thickness at hydrostatic equilibrium and stresses are continuous at the grounding line. In this case, Wilchinsky and Chugunov deduce the continuity of the ice thickness gradient and of the strain rate at the grounding line. They suggest that in order to be physically 
consistent one should find a unique solution of the complete problem describing the ice flow in the stream, transition zone and ice shelf when the horizontal mass flux and bed profile are monotone functions in the main flow direction.

The presence of contact lines between a fluid interface and a solid substrate and the description of their motion constitute a classical problem in fluid mechanics (see for instance [15, 16, 19, 3]) and a challenge both from the mechanical and mathematical points of view. From the mechanical perspective the neighborhood of a moving contact line, if modeled as a Navier-Stokes or Stokes flow, may develop unbounded values of the energy dissipation rate, which is clearly nonphysical. This is known as the contact line paradox in the context of the spreading of a capillary drop over a flat surface. From the mathematical viewpoint, a contact line separates the fluid interface from the solid substrate. At the interface one must impose boundary conditions expressing balance of forces, leading to conditions typically involving first derivatives of the velocity field. On the solid substrate one usually imposes no-slip boundary conditions implying that the velocity field must vanish there.

Solving partial differential equations in domains with boundaries which are either not regular or with mixed boundary conditions is in general a difficult problem since these situations, in general, originate singularities in the solutions (cf. [13, [14, 18, 20]). One is then forced to modify the classical Sobolev or Hölder spaces with suitable weights in order to accommodate such singularities. One must also modify the usual analytical tools (Sobolev embeddings, trace inequalities, etc.) accordingly. In the context of "coating flows", consisting in the motion of a capillary (i.e. under the influence of surface tension) fluid interface, some work has been done towards showing that solutions with moving contact lines do exist (let us mention [25, 26, 22, 9, 10]). The stability of these solutions has been analyzed in [5].

In the present paper the balances of forces at the interface change and therefore the boundary conditions are different. In particular, we cannot count on the regularizing effects of surface tension, a situation that was also present in [21], but in absence of contact lines. This fact gives rise to different singularities at the contact (grounding) line and hence, to different mathematical difficulties. Let us remark finally that the solutions we aim to obtain in this paper are zero contact angle solutions, for which there is a bounded energy dissipation in the vicinity of the contact line.

\subsection{Preliminaries. Equations}

We want to determine whether or not solutions with migrating grounding lines are possible. In order to do that, we make some modeling simplifications. First we assume that the grounding line is moving with constant velocity $U$ in a longitudinal $x$ direction. In a frame of reference attached to the grounding line, this is equivalent to assuming that the solid base is moving with constant velocity $-U$ (see Figure 1). The ice sheet will be modeled as a 2D Stokes flow. In doing this we follow some of the works cited above. In fact, ice is known to be non-Newtonian, with a strain dependent viscosity, in certain regimes, but we will leave the analysis of such "nonlinear" effects for future publications and focus here on the simpler Stokes model. Let us remark in this respect that our study will rely on a variational approach (Section 3) followed by local analysis in the regions of interest and therefore we expect that our results can be extended to the more complex situations involving rheology where variational formulations can be established.

The flow region is given by $D:=\{(x, z): x \in(-M, M), z \in(-1,0)$ if $x<0$ and $z \in$ $(-1+b(x), 0)$ if $x \geqslant 0\}$, where the $x$-coordinate denotes the longitudinal length, i.e. in the direction of the main flow, and the $z$-coordinate denotes the height. The boundary of $D$ consists of three parts: the part $\Gamma_{0}$ in contact with the air, i.e., the surface which we describe by $z=s(x)$ and 
$x \in(-M, M)$ (here we shall assume $s \equiv s(x) \equiv 0$, i.e., a flat surface); the part $\Gamma_{1}$ of the base in contact with the ground, i.e., $\Gamma_{1}:=\{(x, z), x \leqslant 0, z=-1\}$; and the part $\Gamma_{2}$ in contact with the sea, $\Gamma_{2}:=\{(x, z): x \geqslant 0, z=-1+b(x)\} . \Gamma_{2}$ is a free boundary whose shape we wish to determine. The problem is to determin the velocity field $(-U, 0)+\left(v_{x}, v_{z}\right)$, the pressure $\Pi$ and the location and geometry of the free boundaries. Modeling the ice sheet dynamics as a Stokes flow that we assume, without loss of generality, of viscosity $\mu=1$, the equations we shall use are:

- momentum conservation equation: $\nabla \cdot \tau \equiv-\nabla \Pi+\Delta \vec{v}=-\rho_{i} \vec{g}$ in $D$,

- mass conservation equation: $\nabla \cdot \vec{v}=0$ in $D$,

where $\rho_{i}$ is the density of ice and $\tau_{i j}=-\Pi \delta_{i j}+\left(v_{i, j}+v_{j, i}\right)$. The momentum conservation equation may be written, in terms of $p=\Pi-\rho_{i} g(z+1)$, as

$$
\nabla \cdot T=-\nabla p+\Delta \vec{v}=0
$$

where $T$ is the stress tensor,

$$
T_{i j}=-p \delta_{i j}+\left(v_{i, j}+v_{j, i}\right) .
$$

Concerning the boundary conditions, as the upper part of the sheet is in contact with air and we assume that both the normal velocity and the shear stress are zero there, we shall consider the following conditions:

$$
\begin{array}{ll}
\vec{v} \cdot \vec{n}=0 & \text { at } \Gamma_{0}, \\
\vec{t} \tau \vec{n}=\vec{t} T \vec{n}=0 & \text { at } \Gamma_{0},
\end{array}
$$

where $\vec{n}$ and $\vec{t}$ are the normal and tangent vectors to $\Gamma_{0}$ respectively. In the part of the bottom of the ice sheet which is in contact with the solid substrate we impose $\vec{v}=\overrightarrow{0}$, while the other part is in contact with another fluid modeling the sea. We suppose that the second fluid is stationary at all times. Hence we impose as boundary condition between both fluids a balance between viscous stresses on the ice sheet and hydrostatic pressure of the second fluid. Therefore, at the base, depending on whether the ice is grounded or floating over the sea, we consider the following boundary conditions:

$$
\begin{aligned}
\vec{v} & =\overrightarrow{0} \quad \text { on } \Gamma_{1}, \\
\tau \vec{n}=\rho_{w} g b(x) \vec{n} & \Rightarrow T \vec{n}=\rho g b(x) \vec{n} \quad \text { on } \Gamma_{2},
\end{aligned}
$$

where $-\rho_{w} g b(x)$ is the hydrostatic pressure of water in $\Gamma_{2}$ and $\rho=\rho_{w}-\rho_{i}$. We shall also impose inflow and outflow conditions as $x \rightarrow \pm M$ respectively. We denote the inflow as $x \rightarrow-M$ by $\vec{v}^{\text {in }}(z)$ and assume, by compatibility with the boundary conditions, that $\vec{v}^{\text {in }}(-1)=\overrightarrow{0}, v_{z}^{\text {in }}(0)=\overrightarrow{0}$, and the shear stress $\vec{t} T \vec{n}$ is zero at $(-M, 0)$. We may think, for instance, that $\vec{v}^{\text {in }}(z)$ has a parabolic velocity profile, i.e.

$$
\vec{v}^{\text {in }}=\left(\frac{\sigma}{2}\left(1-z^{2}\right), 0\right) .
$$

The outflow $\vec{v}^{\text {out }}(z)$ is such that $v_{z}^{\text {out }}(0)=0$ and the shear stress at $(-M, 0)$ and $(-M,-1)$ is zero. We may think, for instance, of a uniform velocity profile, i.e.

$$
\vec{v}^{\text {out }}=\left(U_{\infty}, 0\right)
$$


Here $\sigma$ and $U_{\infty}$ are two arbitrary positive parameters which are nevertheless linked by the conservation of mass constraint which implies

$$
-U+\int_{-1}^{0} v_{x}^{\text {in }}(z) \mathrm{d} z=-U(1-b(M))+\int_{-1+b(M)}^{0} v_{x}^{\text {out }}(z) \mathrm{d} z .
$$

Notice that the velocity of the grounding line can then be determined from the physically measurable entities $v_{x}^{\text {in }}(z), v_{x}^{\text {out }}(z)$ and $b(M)$ :

$$
U=\frac{1}{b(M)}\left(\int_{-1}^{0} v_{x}^{\text {in }}(z) \mathrm{d} z-\int_{-1+b(M)}^{0} v_{x}^{\text {out }}(z) \mathrm{d} z\right) .
$$

Notice also that the curve $\Gamma_{2}$ may evolve following the velocity field:

$$
\frac{b_{t}}{\left(1+b_{x}^{2}\right)^{1 / 2}}=((-U, 0)+\vec{v}) \cdot \vec{n}=\frac{1}{\left(1+b_{x}^{2}\right)^{1 / 2}}\left[U b_{x}+\left(v_{x}, v_{z}\right)\left(-b_{x}, 1\right)\right] \quad \text { on } \Gamma_{2},
$$

so that the stationary solutions satisfy

$$
0=U b_{x}+v_{z}-b_{x} v_{x} .
$$

A final simplification we make consists in assuming $\Gamma_{0}$ flat, i.e. $\Gamma_{0}=\{(x, 0): x \in[-M, M]\}$ so that the boundary conditions $(1.1)$ and $(1.2)$ become

$$
\begin{array}{ll}
v_{z}=0 & \text { at } \Gamma_{0}, \\
\frac{\partial v_{z}}{\partial x}+\frac{\partial v_{x}}{\partial z}=0 & \text { at } \Gamma_{0} .
\end{array}
$$

Notice that $\nabla \cdot \vec{v}=0$, so we can introduce the stream function $\psi$ such that $\psi_{x}=v_{z}, \psi_{z}=-v_{x}$. Hence, applying the rotation operator to $-\nabla p+\Delta \vec{v}=0$, we arrive at the following biharmonic equation for $\psi$ :

$$
\Delta^{2} \psi=0 \quad \text { in } D .
$$

To summarize, the problem we wish to solve can be formulated in the following terms: find $b(x)$ and a biharmonic function $\psi(x, z)$ in $D$ so that

$$
\begin{array}{ll}
\left(-\psi_{z}, \psi_{x}\right)=\overrightarrow{0} & \text { at } \Gamma_{1}, \\
\vec{n} T \vec{n}=\rho g b(x) & \text { at } \Gamma_{2}, \\
\vec{t} T \vec{n}=0 & \text { at } \Gamma_{2}, \\
U b_{x}+\psi_{x}+b_{x} \psi_{z}=0 & \text { at } \Gamma_{2}, \\
\psi_{x}=\vec{t} T \vec{n}=0 & \text { at } \Gamma_{0}, \\
\left(-\psi_{z}, \psi_{x}\right)=\vec{v}^{\text {in }} & \text { at }(-M, z), \\
\left(-\psi_{z}, \psi_{x}\right)=\vec{v}^{\text {out }} & \text { at }(M, z) .
\end{array}
$$

Condition (1.8) admits a useful representation in terms of the stream function since, by 1.10 ,

$$
\begin{aligned}
b(x) & =-\frac{1}{U} \int_{0}^{x}\left(\psi_{x}+b_{x} \psi_{z}\right) \mathrm{d} x^{\prime}=-\frac{1}{U} \int_{0}^{x} \frac{\mathrm{d}}{\mathrm{d} x^{\prime}} \psi\left(x^{\prime},-1+b\left(x^{\prime}\right)\right) \mathrm{d} x^{\prime} \\
& =-\frac{1}{U}(\psi(x,-1+b(x))-\psi(x,-1)) .
\end{aligned}
$$


From now on we shall take $\psi(x,-1)=0$ for $x \leqslant 0$. Hence we can substitute condition (1.7) by

$$
\psi_{z}=\psi=0 \quad \text { at } \Gamma_{1},
$$

and condition 1.8 by

$$
\vec{n} T \vec{n}=-\gamma \psi \quad \text { at } \Gamma_{2}
$$

where $\gamma=\rho g / U$.

REMARK 1.1 If one considers a fluid with arbitrary viscosity $\mu$ in a domain of arbitrary size $([-M L, 0] \times[-L, 0]) \cup([0, M L] \times[L(-1+b(x)), 0])$, then the problem can be formulated in exactly the same terms as above after the change of variables and unknowns $(x, z) \mapsto(L x, L z)$, $\left(v_{x}, v_{z}\right) \mapsto\left(v_{x} / \mu, v_{z} / \mu\right)$ but with $\gamma=\rho g L^{2} / \mu U$ a dimensionless parameter.

The main result of this paper is the following theorem establishing the existence and uniqueness of solutions to the free boundary problem consisting of $[1.6$ with conditions $(1.9)-11.15)$ :

THEOREM 1.1 If $\vec{v}^{\text {in }}$ and $\vec{v}^{\text {out }}$ are such that $\left\|\vec{v}^{\text {in }}\right\|_{C^{2}[-1,0]},\left\|\vec{v}^{\text {out }}\right\|_{C^{2}[-1+b(M), 0]}$ are small enough and $b(M)$ is also small enough then there exists a unique (weak) solution $\psi \in H^{2+\delta}(D), b \in$ $C^{1+\delta}[0, M](0<\delta \ll 1)$ to the nonlinear free boundary problem given by $[1.6),[1.9]-[1.15$ with $U$ given by 1.5 . Moreover, the asymptotic behavior of the free boundary $b(x)$ near the grounding line located at $(x=0, z=-1)$ is the following:

$$
b(x)=C x^{3 / 2}+o\left(x^{3 / 2}\right) .
$$

The solutions are weak in the sense of solutions obtained via application of the Lax-Milgram theorem (cf. [2] for instance), i.e. solutions of the equivalent weak version of the problem: $A(\omega, \psi)=L \omega$ where $A$ is a bilinear form involving derivatives of $\psi$ only up to second order, and $L$ a linear functional (see Section 3 where these definitions are made rigorous). Analogous results, without smallness conditions, will also be obtained for a linearized version of (1.9)-(1.15) before the proof of Theorem 1.1 .

This paper is organized as follows. In Section 2, we introduce the linearized version of the problem. In Section 3, we prove the existence, uniqueness and partial regularity of solutions. Since the grounding line is a special conflict point, as mixed boundary conditions meet there, we devote most of Section 4 to regularity of the solution in the neighborhood of the grounding line. Regularity of the solution in the rest of the domain is derived from classical results. Next, in Section 5, the representation of the linearized free boundary along with some numerical results is presented and discussed. After solving the linearized problem, in Section 6, we finally address the original nonlinear problem which is treated as a perturbed version of the linearized one, and prove Theorem 1.1. Finally, in an appendix, we deduce some weighed inequalities used in the paper.

\section{The linearized problem}

In our problem, we shall consider $\Gamma_{2}$ as the only free boundary, which is located at $z=-1+b(x)$. In order to deduce a linearized version of the problem when the free boundary is almost flat, in this section we consider $b(x)=\varepsilon h(x)$, with $\varepsilon \ll 1$ and $h(0)=0$. The main difficulty in the analysis lies in the behavior near the grounding line located at $(0,-1)$. We assume that $h_{x}(0)=0$ so that $\Gamma_{1}$ and $\Gamma_{2}$ meet with $C^{1}$ regularity. This hypothesis on the free boundary will be verified a posteriori 
and allows us to perform a diffeomorphism from the domain $D$ where the problem is defined to the strip $S=[-M, M] \times[-1,0]$. In order to do that we introduce a $C^{4}(S)$ cutoff function $\chi(x, z)$ such that

$$
\chi(x, z)= \begin{cases}0 & \text { if } z \geqslant 2 \varepsilon \bar{h}(x), \\ 1 & \text { if } z<\varepsilon \bar{h}(x)\end{cases}
$$

where $\bar{h}$ is a regular function such that $\bar{h}(x)=0$ if $x<0$ and $\bar{h}(x)>h(x)$ if $x \geqslant 0$. Our diffeomorphism is of the form $x^{\prime}=x$ and $z^{\prime}=z-\varepsilon \chi(x, z) h(x)$. Then

$$
\frac{\partial}{\partial x}=\frac{\partial}{\partial x^{\prime}}+(-\varepsilon \chi(x, z) h(x))_{x} \frac{\partial}{\partial z^{\prime}}, \quad \frac{\partial}{\partial z}=\left(1+\varepsilon(\chi(x, z) h(x))_{z}\right) \frac{\partial}{\partial z^{\prime}} .
$$

Analogously one can obtain formulas for higher order derivatives by successive application of 2.1). Next, we shall analyze the boundary conditions. Since $\Gamma_{2}$ is defined by $z=-1+\varepsilon h(x)$, the unit tangent vector is given by

$$
\vec{t}=\left(\frac{1}{\sqrt{1+\varepsilon^{2} h_{x}^{2}}}, \frac{\varepsilon h_{x}}{\sqrt{1+\varepsilon^{2} h_{x}^{2}}}\right)
$$

and the unit normal vector is

$$
\vec{n}=\left(-\frac{\varepsilon h_{x}}{\sqrt{1+\varepsilon^{2} h_{x}^{2}}}, \frac{1}{\sqrt{1+\varepsilon^{2} h_{x}^{2}}}\right) .
$$

Note that we impose as boundary condition between ice shelf and sea water a balance between viscous stresses on the ice and hydrostatic pressure of the sea water and then we need to evaluate $T_{i j} n_{i}$ :

$$
\begin{aligned}
T_{i j} n_{i} & =\left(\begin{array}{cc}
-p+2 \frac{\partial v_{x}}{\partial x} & \frac{\partial v_{x}}{\partial z}+\frac{\partial v_{z}}{\partial x} \\
\frac{\partial v_{x}}{\partial z}+\frac{\partial v_{z}}{\partial x} & -p+2 \frac{\partial v_{z}}{\partial z}
\end{array}\right)\left(\begin{array}{c}
-\frac{\varepsilon h_{x}}{\sqrt{1+\varepsilon^{2} h_{x}^{2}}} \\
\frac{1}{\sqrt{1+\varepsilon^{2} h_{x}^{2}}}
\end{array}\right) \\
& =\frac{1}{\sqrt{1+\varepsilon^{2} h_{x}^{2}}}\left(\begin{array}{c}
\left(-p+2 \frac{\partial v_{x}}{\partial x}\right)\left(-\varepsilon h_{x}\right)+\left(\frac{\partial v_{x}}{\partial z}+\frac{\partial v_{z}}{\partial x}\right) \\
\left(\frac{\partial v_{x}}{\partial z}+\frac{\partial v_{z}}{\partial x}\right)\left(-\varepsilon h_{x}\right)-p+2 \frac{\partial v_{z}}{\partial z}
\end{array}\right) \\
& \approx\left(\begin{array}{c}
\frac{\partial v_{x}}{\partial z}+\frac{\partial v_{z}}{\partial x} \\
-p+2 \frac{\partial v_{z}}{\partial z}
\end{array}\right)+\varepsilon h_{x} \vec{A}\left(D_{x z} v_{j}, p, h_{x}\right) .
\end{aligned}
$$

Then we obtain

$$
\begin{aligned}
& t_{j} T_{i j} n_{i}=\left(\frac{\partial v_{x}}{\partial z}+\frac{\partial v_{z}}{\partial x}\right)+\varepsilon h_{x} C_{1}\left(D_{x z} v_{j}, p, \varepsilon h_{x}\right)=\left(\frac{\partial v_{x}}{\partial z}+\frac{\partial v_{z}}{\partial x}\right)+O(\varepsilon), \\
& n_{j} T_{i j} n_{i}=-p+2 \frac{\partial v_{z}}{\partial z}+\varepsilon h_{x} C_{2}\left(D_{x z} v_{j}, p, \varepsilon h_{x}\right)=-p+2 \frac{\partial v_{z}}{\partial z}+O(\varepsilon) .
\end{aligned}
$$

If we now consider the formulation in terms of the stream function, we arrive at the following equation, to be verified in the interior of our domain $D$ :

$$
\Delta^{2} \psi=\varepsilon F\left(D_{x}^{i} h, D_{x z}^{j} \psi\right), \quad i, j=0, \ldots, 3 .
$$


Noting that $T_{i j} \vec{n}_{j}=\rho g \varepsilon h n_{i}$, we can write the boundary conditions at $\Gamma_{2}$ in the form

$$
\psi_{z z}-\psi_{x x}=\varepsilon h_{x} C_{1}, \quad-p+2 \psi_{x z}=\varepsilon h_{x} C_{2}+\varepsilon \rho g h,
$$

and the kinematic condition as

$$
\varepsilon U h_{x}+\psi_{x}+\varepsilon h_{x} \psi_{z}=0
$$

The nature of the problem allows us to write a system only for $\psi$ by eliminating $p$ from the boundary condition. In order to do that we observe that the tangential component of the vector $\nabla p$ is

$$
\frac{\mathrm{d} p}{\mathrm{~d} \vec{t}}=\vec{t} \cdot \nabla p=\vec{t} \cdot(\Delta \vec{v}) .
$$

Taking into account that

$$
\vec{t} \cdot \Delta \vec{v}=-\Delta \psi_{z}+\varepsilon h_{x} C_{2}^{\prime}\left(D_{x z} v_{j}, p, \varepsilon h_{x}\right)
$$

and taking derivatives in the boundary condition 2.2 - 2 we obtain

$$
-\frac{\mathrm{d} p}{\mathrm{~d} \vec{t}}+2 \frac{\mathrm{d}}{\mathrm{d} \vec{t}}\left(\psi_{x z}\right)=\frac{\mathrm{d}}{\mathrm{d} \vec{t}}\left(\varepsilon h_{x} C_{2}+\varepsilon \rho g h\right) .
$$

If we substitute the expression $(2.4)$ in 2.5 , we obtain

$$
\psi_{z z z}+3 \psi_{x z x}=\varepsilon \rho g h_{x}+\varepsilon h_{x} B_{2}\left(\varepsilon h, \varepsilon h_{x}, \varepsilon h_{x x}, \varepsilon h_{x x x}, D_{x z}^{i} \psi\right), \quad i=1,2,3 .
$$

Finally, from 2.3 we can obtain $\varepsilon h_{x}=-U^{-1}\left(\psi_{x}+\varepsilon h_{x} \psi_{z}\right)$, substitute in 2.6 and conclude

$$
\psi_{z z z}+3 \psi_{x z x}+\gamma \psi_{x}=O(\varepsilon) .
$$

Ignoring terms of order $O(\varepsilon)$, as a result of the change of coordinates and linearization, the same problem is formulated in $S=[-M, M] \times[-1,0]$ as

$$
\begin{array}{rlrl}
\Delta^{2} \psi & =0 & & \text { in } S, \\
\psi_{x}=\psi_{z z}-\psi_{x x}=0 & & \text { on } z=0, x \in[-M, M], \\
\psi=\psi_{z} & =0 & & \text { on } z=-1, x \in[-M, 0], \\
\psi_{z z}-\psi_{x x}=0 & \text { and } \quad 3 \psi_{x x z}+\psi_{z z z}+\gamma \psi_{x}=0 & & \text { on } z=-1, x \in[0, M],
\end{array}
$$

together with the inflow and outflow conditions:

$$
\begin{array}{lll}
\psi_{z}=-v_{x}^{\text {in }}, & \psi_{x}=v_{z}^{\text {in }} & \text { at } x=-M, \\
\psi_{z}=-v_{x}^{\text {out }}, & \psi_{x}=v_{z}^{\text {out }} & \text { at } x=M .
\end{array}
$$

Once a solution $\psi$ is found, one can compute the linearized free boundary $\varepsilon h(x)$ as

$$
\varepsilon h(x)=-\frac{1}{U} \psi(x,-1) .
$$




\section{Existence, uniqueness and partial regularity for the linearized problem}

In this section we will show the existence of a unique weak solution to the linearized system (2.7)(2.12). To this end, we write the linearized system in terms of the stress tensor $T$ defined as

$$
T=\left(\begin{array}{cc}
-p-2 \psi_{x z} & \psi_{x x}-\psi_{z z} \\
\psi_{x x}-\psi_{z z} & -p+2 \psi_{x z}
\end{array}\right)
$$

Therefore we wish to solve:

$$
\begin{aligned}
\nabla \cdot T=0 & \text { in }[-M, M] \times[-1,0], \\
\nabla \cdot \vec{v}=0 & \text { in }[-M, M] \times[-1,0] .
\end{aligned}
$$

The boundary conditions are then

$$
\begin{aligned}
T_{x z}=0, \quad v_{z}=0 & \text { at } z=0, \\
\vec{v}=\overrightarrow{0} & \text { at } z=-1, x<0, \\
T_{x z}=0, \quad T_{z z}=-\gamma \psi=-\gamma \int_{0}^{x} v_{z}(s,-1) & \text { at } z=-1, x>0 .
\end{aligned}
$$

In addition we impose the inflow and outflow boundary conditions as $x \rightarrow \pm M$ : see $(2.11)$ and (2.12). The rest of the section will be devoted to the proof of the following theorem:

THEOREM 3.1 There exists a unique weak solution $\psi \in H^{2}(S)$ to problem 2.7 - 2.10) satisfying (2.11) and 2.12.

Proof. As a first step we write

$$
\vec{v}=\vec{v}^{0}+\vec{v}^{1},
$$

where $\vec{v}^{0}$ is a divergence free $\left[C^{\infty}((-M, M) \times(-1,0))\right]^{2}$ velocity field satisfying the inflow and outflow boundary conditions as well as conditions (3.3), 3.4. Such a velocity field can be constructed, for instance, from a stream function $\psi^{0}$ so that $\vec{v}^{0}=\left(-\psi_{z}^{0}, \psi_{x}^{0}\right)$,

$$
\begin{array}{rcl}
\psi_{x}^{0} \rightarrow v_{z}^{\text {in }}, & \psi_{z}^{0} \rightarrow-v_{x}^{\text {in }} & \text { as } x \rightarrow-M, \\
\psi_{x}^{0} \rightarrow v_{z}^{\text {out }}, & \psi_{z}^{0} \rightarrow-v_{x}^{\text {out }} & \text { as } x \rightarrow+M, \\
& \psi_{x}^{0}, \psi_{z z}^{0}=0 & \text { at } z=0, \\
\psi^{0}, \psi_{z}^{0}=0 & \text { at } z=-1, x<0 .
\end{array}
$$

It is simple to show that there exists a $\psi^{0}$ satisfying these conditions. Consider for instance $\vec{v}^{\text {in }}, \vec{v}^{\text {out }}$ given by 1.3 , 1.4 respectively. Then we can take $\psi^{0}$ as follows:

$$
\psi^{0}=\left(-\frac{\sigma}{3}-\frac{\sigma}{2}\left(z-\frac{z^{3}}{3}\right)\right) \Phi_{1}(x) \eta\left(\frac{z+1}{x}\right)+\left(-\frac{\sigma}{3}-U^{\infty} z\right) \Phi_{2}(x),
$$

where

$$
\eta \in C^{\infty}, \quad \eta(s)= \begin{cases}1 & \text { if } s>1 / 2 M \\ 0 & \text { if } s<1 / 3 M\end{cases}
$$


and $\Phi_{1}(x)+\Phi_{2}(x)=1$ with

$$
\begin{aligned}
& \Phi_{1} \in C^{\infty}, \quad \Phi_{1}(x)= \begin{cases}1 & \text { if }-M \leqslant x<0, \\
0 & \text { if } x \geqslant \delta,\end{cases} \\
& \Phi_{2} \in C^{\infty}, \quad \Phi_{2}(x)= \begin{cases}0 & \text { if } x \leqslant 0, \\
1 & \text { if } \delta<x \leqslant M,\end{cases}
\end{aligned}
$$

and $\delta=M / 10$. Then $\psi^{0} \in C^{\infty}((-M, M) \times(-1,0))$ and satisfies 3.6 - 3.9. Notice, in particular, that

$$
c_{1}(x) \equiv \psi_{z z}^{0}-\psi_{x x}^{0} \quad \text { and } \quad c_{2}(x) \equiv \gamma \psi_{x}^{0}+\left(\psi_{z z z}^{0}+3 \psi_{z x x}^{0}\right)
$$

are bounded at $z=-1, x>0$. The velocity $\vec{v}^{1}$ and its associated stress tensor $T^{1}$ will satisfy the system

$$
\begin{array}{ll}
\nabla \cdot T^{1}=\vec{s}_{1} & \text { in }[-M, M] \times[-1,0], \\
\nabla \cdot \vec{v}^{1}=0 & \text { in }[-M, M] \times[-1,0],
\end{array}
$$

with $\overrightarrow{s_{1}}$ (depending on $\psi^{0}$ ) bounded. The boundary conditions are then

$$
\begin{gathered}
T_{x z}^{1}=0, \quad v_{z}^{1}=0 \quad \text { at } z=0, \\
\vec{v}^{1}=\overrightarrow{0} \quad \text { at } z=-1, x<0, \\
T_{x z}^{1}=-c_{1}(x), \quad T_{z z}^{1}(x,-1)+\gamma \int_{0}^{x} v_{z}^{1}(s,-1)=-\int_{0}^{x} c_{2}(s) \mathrm{d} s \quad \text { at } z=-1, x>0,
\end{gathered}
$$

together with the conditions that the stream function associated to $\vec{v}^{1}$, i.e. the function $\psi^{1}$ such that $\vec{v}^{1}=\left(-\psi_{z}^{1}, \psi_{x}^{1}\right)$, vanishes together with its first derivatives at $x= \pm M$. We introduce as a set of test functions the space $X$ which is the closure with respect to the norm

$$
\|\vec{\varphi}\|_{X} \equiv\left(\int_{S}|\nabla \vec{\varphi}|^{2}\right)^{1 / 2}
$$

of the set

$$
\begin{aligned}
\left\{\vec{\varphi}=\left(\varphi_{1}, \varphi_{2}\right) \in\left[C_{0}^{\infty}(\bar{S})\right]^{2}:\right. & \nabla \cdot \vec{\varphi}=0, \\
& \varphi_{2}(x, 0)=0, x \in[-M, M], \\
& \varphi_{1}(x,-1)=\varphi_{2}(x,-1)=0, x \in[-M, 0], \\
& \vec{\varphi}=0 \text { at } x= \pm M\} .
\end{aligned}
$$

In terms of the stream function, we will work with the set of test functions formed by those $\omega$ such that $\vec{\varphi}=\left(-\omega_{z}, \omega_{x}\right)$ is in $X$. Korn's inequality which applies to bounded domains and for functions $\vec{\varphi}$ vanishing at a part of the boundary (see [23]) and Sobolev embeddings imply that this set $Y$ is the closure with respect to the $H^{2}(S)$ norm of the set of stream functions whose associated velocities belong to $X$. More precisely,

$$
\begin{aligned}
Y=\left\{\omega \in H^{2}(S):\right. & \omega=0 \text { on }([-M, M], 0), \\
& \omega=\omega_{z}=0 \text { on }([-M, 0],-1), \\
& \left.\omega_{x}=\omega=0 \text { on }( \pm M,[-1,0])\right\} .
\end{aligned}
$$


We multiply equation 3.10 by $\vec{\varphi} \in X$, integrate by parts and obtain

$$
\int_{S} \vec{\varphi} \operatorname{div} T^{1}=-\frac{1}{2} \int_{S}\left(\varphi_{i, j}+\varphi_{j, i}\right)\left(v_{i, j}^{1}+v_{j, i}^{1}\right)+\int_{\partial S} \vec{\varphi} T^{1} \vec{n}=\int_{S} \vec{\varphi} \cdot \overrightarrow{s_{1}} .
$$

Hence,

$$
\frac{1}{2} \int_{S}\left(\varphi_{i, j}+\varphi_{j, i}\right)\left(v_{i, j}^{1}+v_{j, i}^{1}\right)=\int_{\partial S} \vec{\varphi} T^{1} \vec{n}-\int_{S} \vec{\varphi} \cdot \overrightarrow{s_{1}} .
$$

Set $L=[-M, M]$ and $L^{+}=[0, M]$. Given the boundary conditions imposed on $T^{1}$ and the properties of $X$ we can compute

$$
\begin{aligned}
\int_{\partial S} \vec{\varphi} T^{1} \vec{n} & =\int_{L, z=0}\left(\varphi_{1} T_{x z}^{1}+\varphi_{2} T_{z z}^{1}\right)-\int_{L, z=-1}\left(\varphi_{1} T_{x z}^{1}+\varphi_{2} T_{z z}^{1}\right) \\
& =\int_{L^{+}, z=-1}^{x}\left(c_{1}(x) \varphi_{1}(x,-1)+\left[\gamma\left(\int_{0}^{x} v_{z}^{1}(s,-1)\right)+\int_{0}^{x} c_{2}(s) \mathrm{d} s\right] \varphi_{2}(x,-1)\right) .
\end{aligned}
$$

Hence we arrive at the formula

$$
\begin{aligned}
& \frac{1}{2} \int_{S}\left(\varphi_{i, j}+\varphi_{j, i}\right)\left(v_{i, j}^{1}+v_{j, i}^{1}\right)-\gamma \int_{L^{+}, z=-1}\left(\int_{0}^{x} v_{z}^{1}(s,-1)\right) \varphi_{2}(x,-1) \\
&=\int_{L^{+}, z=-1} c_{1}(x) \varphi_{1}(x,-1)+\int_{L^{+}, z=-1}\left(\int_{0}^{x} c_{2}(s) \mathrm{d} s\right) \varphi_{2}(x,-1)-\int_{S} \vec{\varphi} \cdot \overrightarrow{s_{1}} .
\end{aligned}
$$

Next we write the expression above in terms of the stream function $\omega$ of $\vec{\varphi}$ and $\psi$ of $\vec{v}^{1}$ assuming $\omega \in Y$ :

$$
\begin{aligned}
a(\omega, \psi) & -\gamma \int_{L^{+}, z=-1} \psi(x,-1) \omega_{x}(x,-1) \\
= & -\int_{L^{+}, z=-1} c_{1}(x) \omega_{z}(x,-1)+\int_{L^{+}, z=-1} c_{2}(x) \omega_{x}(x,-1)-\int_{S}\left(-\omega_{z}, \omega_{x}\right) \cdot \overrightarrow{s_{1}},
\end{aligned}
$$

where

$$
a(\omega, \psi)=\frac{1}{2} \int_{S}\left(\varphi_{i, j}+\varphi_{j, i}\right)\left(v_{i, j}^{1}+v_{j, i}^{1}\right) .
$$

Equation 3.11 can be written symbolically as

$$
A(\omega, \psi)=L \omega
$$

where

$$
A(\omega, \psi) \equiv a(\omega, \psi)-b(\omega, \psi) \quad \text { and } \quad b(\omega, \psi) \equiv \gamma \int_{L^{+}, z=-1} \psi(x,-1) \omega_{x}(x,-1)
$$

are bilinear forms continuous in $Y$ (for $a(\cdot, \cdot)$ this is obvious while for $b(\cdot, \cdot)$ it is due to the fact that the trace of functions in $H^{2}(S)$ lies in $H^{1}(\partial S)$ ) and

$$
L \omega \equiv-\int_{L^{+}, z=-1} c_{1}(x) \omega_{z}(x,-1)+\int_{L^{+}, z=-1} c_{2}(x) \omega(x,-1)-\int_{S}\left(-\omega_{z}, \omega_{z}\right) \cdot \vec{s}_{1}
$$

is a continuous linear functional on $Y$ given the boundedness of $c_{1}, c_{2}$ and $\vec{s}_{1}$ and the fact that the 
trace of $H^{2}$ is in $H^{1}$ in a bounded domain in two dimensions. Finally, $A(\omega, \psi)$ is coercive since

$$
A(\omega, \omega) \equiv a(\omega, \omega)-\gamma \int_{L^{+}, z=-1} \omega(x,-1) \omega_{x}(x,-1)=a(\omega, \omega) \geqslant C\|\omega\|_{H^{2}}
$$

We have used, for the last inequality, Korn's inequality which allows us estimate the norm of any $\varphi_{i, j}$ in $L^{2}$ by $a(\omega, \omega)$, and Poincaré's inequality which allows estimating any $\varphi_{i}$ in $L^{2}$ by the norms of $\varphi_{i, j}$ in $L^{2}$ as well as the norm of $\omega$ in $L^{2}$. These inequalities are valid in bounded domains with Lipschitz boundaries if $\vec{\varphi}$ and $\omega$ vanish at a part of the boundary, as is the case here. Lax-Milgram's theorem then implies the existence of a unique solution to our problem such that $\psi \in H^{2}(S)$.

\section{Regularity in the neighborhood of the grounding line}

In order to study the regularity we shall follow the classical approach except for the conflict point $(x=0, z=-1)$, where mixed boundary conditions meet and the solutions might develop some kind of singularities. We introduce suitable cut-off functions to isolate different regions (see Figure 2). More precisely, we consider $\chi_{i}, i=1, \ldots, 5$, such that $\chi_{i} \in C^{\infty}(S), \sum \chi_{i}=1$,

$$
\begin{aligned}
& \operatorname{supp}\left(\chi_{1}\right)=\Omega_{1} \subset[-M, M] \times(-3 / 4,-1 / 4), \\
& \operatorname{supp}\left(\chi_{2}\right)=\Omega_{2} \subset[-M, M] \times(-1 / 2,0), \\
& \operatorname{supp}\left(\chi_{3}\right)=\Omega_{3} \subset([-1, M] \times[-1,-1 / 2]) \backslash B_{\delta / 2}(0,-1), \\
& \operatorname{supp}\left(\chi_{4}\right)=\Omega_{4} \subset([-M, 1] \times[-1,-1 / 2]) \backslash B_{\delta / 2}(0,-1), \\
& \operatorname{supp}\left(\chi_{5}\right)=\Omega_{5}=B_{\delta}(0,-1) \cap S,
\end{aligned}
$$

and

$$
\chi_{5}=1 \quad \text { in } B_{\delta / 2}(0,-1) \cap S .
$$

Then we can write the stream function $\psi$ in the following way:

$$
\psi=\sum_{i=1}^{5} \psi_{i} \quad \text { with } \psi_{i}=\chi_{i} \psi, i=1, \ldots, 5 .
$$

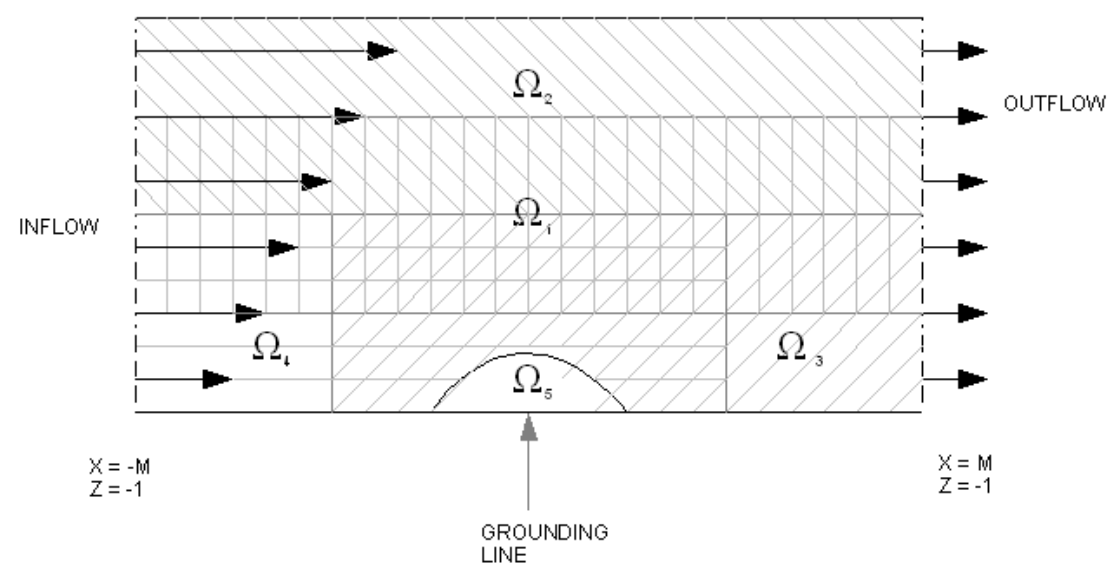

FIG. 2. Illustration of the partition of the domain used to study the regularity of the solution. 
In the previous sections we obtained $\psi \in H^{2}$ but we expect the solution to have a much better regularity locally in the neighborhood of any point in $S$ given the elliptic nature of the system (cf. [1]). The only exception to this is the region $\Omega_{5}$ where mixed boundary conditions have to be imposed and this fact, as mentioned above, may give rise to singularities that we wish to understand in the next sections. The analysis of the regularity for $\psi_{i}, i=1,2,3,4$, follows the lines of [9, 10], where almost identical analysis was developed in full detail. The only essential difference is in the boundary condition $(2.10)$ for $\psi_{3}$ which was slightly different: $3 \psi_{3, x x z}+\psi_{3, z z z}+\gamma \psi_{3, x x x}=0$. Making $\gamma=0$ in both problems leads to the same system. In their case, this particular choice does not affect the regularity outside $\Omega_{5}$ and in our case, the term $\gamma \psi_{3, x}$ dropped out is more regular than the other terms in the boundary condition and its presence does not affect regularity. The conclusion then is $C^{4}$ regularity for $\psi$ inside $S$ and outside $\Omega_{5}$ as in [9, 10]. If we assume that $\chi_{5}$ depends only on $r$, the distance to $(0,-1)$, then using the system 2.7$)-2.10$ ) one can write the following system for $\psi_{5}$ :

$$
\begin{aligned}
\Delta^{2} \psi_{5}=J\left(\psi, D \psi, D^{2} \psi, D^{3} \psi\right) & \text { in } S, \\
\psi_{5}=0 & \text { at } x<0, z=-1, \\
\psi_{5, z}=0 & \text { at } x<0, z=-1, \\
\psi_{5, z z}-\psi_{5, x x}=B_{1}(\psi, D \psi) & \text { at } x>0, z=-1, \\
3 \psi_{5, x x z}+\psi_{5, z z z}=-\gamma \chi_{5} \psi_{x}+B_{2}\left(\psi, D \psi, D^{2} \psi\right) & \text { at } x>0, z=-1,
\end{aligned}
$$

where

$$
\begin{aligned}
B_{1}(\psi, D \psi)= & 2 \chi_{5, z} \psi_{z}+\chi_{5, z z} \psi-2 \chi_{5, x} \psi_{x}-\chi_{5, x x} \psi, \\
B_{2}\left(\psi, D \psi, D^{2} \psi\right)= & 3\left(\chi_{5, z x x} \psi+2 \chi_{5, z x} \psi_{x}+\chi_{5, z} \psi_{x x}+\chi_{5, x x} \psi_{z}+2 \chi_{5, x} \psi_{z x}\right) \\
& +\left(\chi_{5, z z z} \psi+3 \chi_{5, z} \psi_{z z}+3 \chi_{5, z z} \psi_{z}\right) .
\end{aligned}
$$

The function $J\left(\psi, D \psi, D^{2} \psi, D^{3} \psi\right)$ and both $B_{1}(\psi, D \psi)$ and $B_{2}\left(\psi, D \psi, D^{2} \psi\right)$ are compactly supported away from $(x, z)=(0,-1)$. From the classical results of Agmon, Douglis and Nirenberg [1] and the discussion above it follows that $\psi \in C^{4}\left(\{(-M, M) \times[-1,0]\} \backslash B_{\delta / 2}(0,-1)\right)$ so that $J\left(\psi, D \psi, D^{2} \psi, D^{3} \psi\right), B_{1}(\psi, D \psi), B_{2}\left(\psi, D \psi, D^{2} \psi\right)$ are bounded. Therefore $\psi_{5}$ satisfies

$$
\begin{aligned}
\Delta^{2} \psi_{5}=J(x, y) z & \text { in } \mathbb{R} \times[-1,+\infty], \\
\psi_{5}=0 & \text { at } x<0, z=-1, \\
\psi_{5, z}=0 & \text { at } x<0, z=-1, \\
\psi_{5, z z}-\psi_{5, x x}=G(x) & \text { at } x>0, z=-1, \\
3 \psi_{5, x x z}+\psi_{5, z z z}=F(x) & \text { at } x>0, z=-1,
\end{aligned}
$$

where

$$
\begin{aligned}
& G(x)=B_{1}(\psi, D \psi), \\
& F(x)=-\gamma \chi_{5} \psi_{x}+B_{2}\left(\psi, D \psi, D^{2} \psi\right) .
\end{aligned}
$$

Notice that both $G$ and $F$ are in $L^{2}\left(\mathbb{R}^{+}\right), J \in L^{2}\left(\mathbb{R}^{+} \times[-1,+\infty]\right)$ and all of these functions are compactly supported away from $(0,-1)$. We can express the solution for $44.6-(4.10)$ as $\psi_{5}=$ 
$\psi_{5}^{(1)}+\psi_{5}^{(2)}$ where $\psi_{5}^{(1)}$ solves $4.6-4.10$ with homogeneous boundary conditions and $\psi_{5}^{(2)}$ solves

$$
\begin{aligned}
\Delta^{2} \psi_{5}^{(2)}=0 & \text { in } \mathbb{R} \times[-1,+\infty], \\
\psi_{5}^{(2)}=0 & \text { at } x<0, z=-1, \\
\psi_{5, z}^{(2)}=0 & \text { at } x<0, z=-1, \\
\psi_{5, z z}^{(2)}-\psi_{5, x x}^{(2)}=G(x) & \text { at } x>0, z=-1, \\
3 \psi_{5, x x z}^{(2)}+\psi_{5, z z z}^{(2)}=F(x) & \text { at } x>0, z=-1 .
\end{aligned}
$$

The function $\psi_{5}^{(1)}$ can be written in terms of Green's function for the biharmonic operator with homogeneous boundary conditions. This operator can be constructed straightforwardly by using Mellin transforms. Since the source $J(x, y)$ is compactly supported away from $(0,-1)$ it is a simple matter to find that $\psi_{5}^{(1)}$ behaves in the neighborhood of $(0,-1)$ as a solution to the homogeneous version of 4.6-4.10 which is locally in $H^{2}$ (see for instance Maz'ya et al. [17, Chapters 6 and 7], where this fact is established rigorously). Using polar coordinates around $(0,-1)$ the problem can be written in the form

$$
\begin{aligned}
& \Delta^{2} \psi=\left(\frac{\partial^{2}}{\partial r^{2}}+\frac{1}{r} \frac{\partial}{\partial r}+\frac{1}{r^{2}} \frac{\partial^{2}}{\partial^{2} \theta}\right)^{2} \psi=0, \\
& \psi=0 \quad \text { at } \theta=\pi, \\
& \psi_{\theta}=0 \quad \text { at } \theta=\pi, \\
& \psi_{r r}-\frac{1}{r^{2}} \psi_{\theta \theta}-\frac{1}{r} \psi_{r}=0 \quad \text { at } \theta=0, \\
& -\frac{1}{r^{3}} \psi_{\theta \theta \theta}-\frac{3}{r} \psi_{r r \theta}+\frac{3}{r^{2}} \psi_{r \theta}-\frac{4}{r^{3}} \psi_{\theta}=0 \quad \text { at } \theta=0,
\end{aligned}
$$

and the solutions can be found in the form

$$
\psi(r, \theta)=r^{\alpha+1} f_{\alpha}(\theta)
$$

where

$$
f_{\alpha}(\theta)=A \sin ((\alpha+1) \theta)+B \cos ((\alpha+1) \theta)+C \sin ((\alpha-1) \theta)+D \cos ((\alpha-1) \theta) .
$$

The coefficients $A, B, C$ and $D$ should be determined by the boundary conditions, and $\alpha$ chosen in such a way that they are satisfied. In fact, considering (4.16) and imposing boundary conditions, we get a homogeneous system for $A, B, C$ and $D$ which will have a nontrivial solution only if the determinant of the matrix of the system written in terms of $\alpha, \sin ((\alpha+1) \pi)$ and $\cos ((\alpha+1) \pi)$ is null. The determinant is

$$
16(\alpha-1) \alpha^{4} \cos ^{2}((\alpha+1) \pi)
$$

so that the only possible solutions are $\alpha=0, \alpha=1$ and the solutions of

$$
\cos ^{2}((\alpha+1) \pi)=0, \quad \text { i.e. } \quad(\alpha+1) \pi=(n+1 / 2) \pi, n \in \mathbb{Z} .
$$

It can be easily shown that the roots $\alpha=0$ and $\alpha=1$ are such that the solution (4.17) degenerates and an analysis of these two specific cases leads to the nonexistence of solutions satisfying all the 
boundary conditions. Therefore $\alpha=n-1 / 2$. Taking into consideration that $\psi \in H_{\mathrm{loc}}^{2}\left(B_{R}(-1,0)\right)$, we find that the first value of $\alpha$ compatible with it is $\alpha=1 / 2$. This implies that the $H^{2}$ solution should be such that

$$
\psi_{5}^{(1)} \sim C r^{3 / 2} f_{1 / 2}(\theta)+o\left(r^{2}\right) \quad \text { as } r \rightarrow 0 .
$$

\subsection{Analysis of problem 4.11 -4.15)}

Next we will study the behavior of the solutions $\psi_{5}^{(2)}$ of $4.11-4.15$ in the neighborhood of the grounding line. For simplicity of notation we will denote $\psi_{5}^{(2)}$ by $\psi$. Notice that $\psi \in$ $H^{2}\left(B_{R}(-1,0)\right)$ and supp $\psi \subset \Omega_{5} \equiv B_{R}(-1,0)$ for $R>\delta$. Then, if we introduce polar coordinates $(r, \theta)$ around $(0,-1)$ and use Poincaré's inequality it is straightforward to prove the following equivalence of norms:

$$
\|\psi\|_{H^{2}\left(B_{R}(-1,0)\right)} \sim \int_{0}^{\pi} \int_{0}^{R}\left[\sum_{i=0}^{2}\left|\frac{1}{r^{2}} \frac{\partial^{i} \psi}{\partial \theta^{i}}\right|^{2}+\sum_{i=0}^{1}\left|\frac{1}{r} \frac{\partial}{\partial r} \frac{\partial^{i} \psi}{\partial \theta^{i}}\right|^{2}+\left|\frac{\partial^{2} \psi}{\partial r^{2}}\right|^{2}\right] r \mathrm{~d} r \mathrm{~d} \theta
$$

The homogeneity in $r$ of the above expression suggests the introduction of the following Mellin transform in $r$ of $\psi$ :

$$
\tilde{\psi}(\lambda, \theta)=\int_{0}^{\infty} \psi(r, \theta) r^{i \lambda-2} \mathrm{~d} r
$$

in terms of which one can write

$$
\|\psi\|_{H^{2}\left(B_{R}(-1,0)\right)} \sim \int_{-\infty}^{\infty} \int_{0}^{\pi} \sum_{i=0}^{2}\left|\frac{\partial^{i} \tilde{\psi}}{\partial \theta^{i}}(\lambda, \theta)\right|^{2} \mathrm{~d} \lambda \mathrm{d} \theta .
$$

In general, one can define the $\alpha$-Mellin transform as follows:

Definition 4.1 Given a function $f \in L^{2}\left([0, \infty), u^{2 \alpha}\right)$, its $\alpha$-Mellin transform is given by

$$
\tilde{f}^{\alpha}(\lambda)=\int_{0}^{\infty} f(u) u^{i \lambda+\alpha-1 / 2} \mathrm{~d} u,
$$

where $\lambda \in \mathbb{R}$. The corresponding inverse Mellin transform is given by

$$
f(u)=\frac{1}{2 \pi} \int_{-i \infty}^{i \infty} \tilde{f}^{\alpha}(\lambda) u^{-i \lambda-\alpha-1 / 2} \mathrm{~d} \lambda .
$$

Note that by integration by parts,

$$
\tilde{f}_{x}^{\alpha}=-(i \lambda-1 / 2+\alpha) \tilde{f}^{\alpha-1} .
$$

In order to study regularity near the corner, we shall make use of the a priori information on the regularity of weak solutions obtained in Section 3 . More precisely, we shall write the boundary conditions 4.14, 4.15) in the form

$$
\begin{aligned}
\psi_{z z}-\psi_{x x} & =G(x), \\
\psi_{z z z}+3 \psi_{x z x} & =F(x),
\end{aligned}
$$


and we shall solve the system with the right hand sides of (4.20), (4.21) being general functions $G$ and $F$ belonging to $L^{2}(\mathbb{R})$, as dictated by the partial regularity result obtained in Section 3 In order to study the asymptotic behavior of the stream function at the grounding line point we follow the same procedure performed in [5]. First we write the problem we have in $\Omega_{5}$ in terms of polar coordinates $(r, \theta)$. The equation in the interior is

$$
\Delta^{2} \psi=\left(\frac{\partial^{2}}{\partial r^{2}}+\frac{1}{r} \frac{\partial}{\partial r}+\frac{1}{r^{2}} \frac{\partial^{2}}{\partial^{2} \theta}\right)^{2} \psi=0
$$

and the boundary conditions are:

$$
\begin{aligned}
& \psi=0 \quad \text { at } \theta=\pi, \\
& \psi_{\theta}=0 \quad \text { at } \theta=\pi, \\
& \psi_{r r}-\frac{1}{r^{2}} \psi_{\theta \theta}-\frac{1}{r} \psi_{r}=G \quad \text { at } \theta=0, \\
& -\frac{1}{r^{3}} \psi_{\theta \theta \theta}-\frac{3}{r} \psi_{r r \theta}+\frac{3}{r^{2}} \psi_{r \theta}-\frac{4}{r^{3}} \psi_{\theta}=F \quad \text { at } \theta=0 .
\end{aligned}
$$

Given the exponent in the Mellin transform chosen in the definition (4.19), we multiply the above equations by $r^{i \lambda+2}, r^{i \lambda-2}, r^{i \lambda-2}, r^{i \lambda}$ and $r^{i \lambda+1}$ respectively. Next we integrate in $r$ from 0 to infinity and after some integrations by parts we obtain:

$$
\begin{array}{rlrl}
{\left[(i \lambda+1)^{2}+\frac{\partial^{2}}{\partial \theta^{2}}\right]\left[(i \lambda-1)^{2}+\frac{\partial^{2}}{\partial \theta^{2}}\right] \tilde{\psi}} & =0, & \\
\tilde{\psi}=0 & \text { at } \theta=\pi, \\
\frac{\partial}{\partial \theta} \tilde{\psi}=0 & \text { at } \theta=\pi, \\
{\left[-\frac{\partial^{2}}{\partial \theta^{2}}+(i \lambda+1)(i \lambda-1)\right] \tilde{\psi}=\tilde{G}(\lambda)} & \text { at } \theta=0, \\
{\left[-\frac{\partial^{3}}{\partial \theta^{3}}-(3(i \lambda+1)(i \lambda-1)+4) \frac{\partial}{\partial \theta}\right] \tilde{\psi}=\tilde{F}(\lambda)} & \text { at } \theta=0,
\end{array}
$$

where $\tilde{\psi}(\lambda, \theta)$ is the Mellin transform of $\psi(r, \theta)$ as defined in 4.19 . The solution of the equation (4.22) is of the form

$$
\tilde{\psi}^{a}(\lambda, \theta)=A \cos ((i \lambda-1) \theta)+B \sin ((i \lambda-1) \theta)+C \cos ((i \lambda+1) \theta)+D \sin ((i \lambda+1) \theta) .
$$

The coefficients $A, B, C, D$ are functions of $\lambda$ which are determined by the boundary conditions. Let us use the following notation: $b=i \lambda-1, s=\sin ((i \lambda-1) \pi), c=\cos ((i \lambda-1) \pi)$ and $\gamma=2 b(b+1)(b+2)$. Then

$$
\left(\begin{array}{cccc}
c & s & c & s \\
-b s & b c & -(b+2) s & (b+2) c \\
2 b & 0 & -2(b+2) & 0 \\
0 & -2 \gamma & 0 & 2 \gamma
\end{array}\right)\left(\begin{array}{l}
A \\
B \\
C \\
D
\end{array}\right)=\left(\begin{array}{c}
0 \\
0 \\
\tilde{G}(\lambda) \\
\tilde{F}(\lambda)
\end{array}\right)
$$


where

$$
\tilde{G}(\lambda)=\int_{0}^{\infty} G(r) r^{i \lambda} \mathrm{d} r \quad \text { and } \quad \tilde{F}(\lambda)=\int_{0}^{\infty} F(r) r^{i \lambda+1} \mathrm{~d} r .
$$

Next, we find that

$$
\left(\begin{array}{l}
A \\
B \\
C \\
D
\end{array}\right)=\left(\begin{array}{cccc}
\frac{b+2}{2 c(b+1)} & 0 & \frac{1}{4(b+1)} & \frac{s(b+2)}{4 c \gamma(b+1)} \\
\frac{-b(b+2) s}{2 c^{2}(b+1)} & \frac{-1}{2 c} & \frac{s}{4 c(b+1)} & \frac{-\left(c^{2} b^{2}+3 c^{2} b+2 c^{2}+s^{2} b^{2}+2 s^{2} b\right)}{4 \gamma c^{2}(b+1)} \\
\frac{b}{2 c(b+1)} & 0 & \frac{-1}{4(b+1)} & \frac{s b}{4 c \gamma(b+1)} \\
\frac{b(b+2) s}{2 c(b+1)} & \frac{1}{2 c} & \frac{-s}{4 c(b+1)} & \frac{b\left(c^{2} b+c^{2}+s^{2} b+2 s^{2}\right)}{4 \gamma c^{2}(b+1)}
\end{array}\right)\left(\begin{array}{c}
0 \\
0 \\
\tilde{G}(\lambda) \\
\tilde{F}(\lambda)
\end{array}\right) .
$$

We are particularly interested in the values for $\theta=0$. Hence,

$$
\begin{aligned}
\tilde{\psi}(\lambda, 0)=A+C & =\left(\frac{s(b+2)}{4 c \gamma(b+1)}+\frac{s b}{4 c \gamma(b+1)}\right) \tilde{F}(\lambda) \\
& =\frac{2 s}{4 c \gamma} \tilde{F}(\lambda)=\frac{\tan ((i \lambda-1) \pi)}{2 \gamma} \tilde{F}(\lambda),
\end{aligned}
$$

and therefore,

$$
\tilde{\psi}(\lambda, 0)=\frac{\tan ((i \lambda-1) \pi)}{2 \gamma} \tilde{F}(\lambda)
$$

Note that

$$
b(b+1)(b+2) \tilde{\psi}=-\tilde{\psi}_{r r r}^{3 / 2},
$$

where

$$
\tilde{\psi}_{r r r}^{3 / 2}=\int_{0}^{\infty} \psi_{r r r}(r) r^{i \lambda+1} \mathrm{~d} r
$$

Hence,

$$
\tilde{\psi}_{r r r}^{3 / 2}=-\frac{\tilde{F}(\lambda)}{2} \tan ((i \lambda-1) \pi) .
$$

Next, we shall use the inversion formula to obtain

$$
\psi_{r r r}(r, \theta=0)=-\frac{1}{2 \pi} \frac{1}{r^{2}} \int_{-\infty}^{\infty} \mathrm{d} \lambda r^{-i \lambda} \tilde{F}(\lambda) \tan ((i \lambda-1) \pi),
$$

or, computing the $\lambda$ integral as in [5],

$$
\psi_{r r r}(r, \theta=0)=-\frac{1}{2 \pi} \frac{1}{r^{3 / 2}} \text { P. V. } \int_{0}^{\infty} \frac{\xi^{3 / 2}}{r-\xi} f(\xi) \mathrm{d} \xi .
$$

Then

$$
\psi_{r r r}=\psi_{r r r, 1}+\psi_{r r r, 2},
$$

where

$$
\begin{aligned}
& \psi_{r r r, 1}=-\frac{1}{2 \pi} \frac{1}{r^{1 / 2}} \text { P. V. } \int_{0}^{\infty} \frac{\xi^{1 / 2}}{r-\xi} f(\xi) \mathrm{d} \xi \\
& \psi_{r r r, 2}=\frac{1}{2 \pi} \frac{1}{r^{3 / 2}} \int_{0}^{\infty} \xi^{1 / 2} f(\xi) \mathrm{d} \xi
\end{aligned}
$$


Notice now that, given that the support of $f(\xi)$ is bounded, one can estimate

$$
\int_{0}^{\infty} \xi^{1 / 2}|f(\xi)| \mathrm{d} \xi \leqslant C\|f\|_{L^{2}} .
$$

On the other hand, we can estimate 4.23] using Lemma 2.2 from [5] to conclude

$$
\int_{-\infty}^{\infty}\left|\tilde{\psi}_{r r r, 1}^{s}\right|^{2} \mathrm{~d} \lambda \leqslant C \int_{-\infty}^{\infty}\left|\tilde{f}^{s}\right|^{2} \mathrm{~d} \lambda, \quad 0<s<1(s \neq 1 / 2),
$$

so that Plancherel's identity for Mellin transforms yields

$$
\left\|r^{s} \psi_{r r r, 1}(r)\right\|_{L^{2}}^{2}=\int_{0}^{\infty} \xi^{2 s}\left|\psi_{r r r, 1}(\xi)\right|^{2} \mathrm{~d} \xi \leqslant C \int_{0}^{\infty} \xi^{2 s}|f(\xi)|^{2} \mathrm{~d} \xi \leqslant C\|f\|_{L^{2}}^{2}
$$

since $f(\xi)$ is compactly supported. Hence, by the Hardy type inequality proved in Lemma 7.1 in the Appendix,

$$
\frac{\left|\psi_{r r, 1}(r, 0)-\psi_{r r, 1}(0,0)\right|}{r^{1 / 2-s}} \leqslant\left\|r^{s} \psi_{r r r, 1}\right\|_{L^{2}} \leqslant C\|f\|_{L^{2}} .
$$

Equations 4.23, 4.24) and inequality 4.25) imply that

$$
\psi_{r r}(r, 0)=\frac{C}{r^{1 / 2}}+O(1) \quad \text { as } r \rightarrow 0,
$$

and hence,

$$
\psi_{r}(r, 0)=2 C r^{1 / 2}+O(r) \quad \text { as } r \rightarrow 0 .
$$

REMARK 4.1 The first order term of the representation of the free boundary is given, according to expressions 2.13, 4.18 and 4.26, by

$$
b(x)=\varepsilon h(x) \sim C x^{3 / 2} .
$$

\section{Representation of the linearized free boundary and numerical approximations}

In this section we shall show that the solution to the linearized problem admits a representation as a power series analytical in $\gamma$. More precisely, one has:

THEOREM 5.1 If $\gamma<\gamma_{0}$ for $\gamma_{0}$ small enough then the solution to

$$
\begin{aligned}
& \Delta^{2} \psi=0 \quad \text { in } S, \\
& \psi=\psi_{z z}-\psi_{x x}=0 \quad \text { on } z=0, x \in[-M, M] \text {, } \\
& \psi=\psi_{z}=0 \quad \text { on } z=-1, x \in[-M, 0], \\
& \psi_{z z}-\psi_{x x}=0 \text { and } 3 \psi_{x x z}+\psi_{z z z}+\gamma \psi_{x}=0 \quad \text { on } z=-1, x \in[0, M] \text {, }
\end{aligned}
$$

together with the inflow and outflow conditions

$$
\begin{array}{lll}
\psi_{z}=-v_{x}^{\text {in }}, & \psi_{x}=v_{z}^{\text {in }} & \text { at } x=-M, \\
\psi_{z}=-v_{x}^{\text {out }}, & \psi_{x}=v_{z}^{\text {out }} & \text { at } x=M,
\end{array}
$$


can be written as

$$
\psi=\sum_{n=0}^{\infty} \gamma^{n} \psi^{(n)},
$$

where $\left\|\psi^{(n)}\right\|_{H^{2}(S)} \leqslant C_{0} C^{n}$ for some constant $C$ that depends only on $M$.

Proof. We decompose our problem into two: the problem $P_{0}$ which is $5.1-(5.6)$ with $\gamma=0$ and whose solution we call $\psi^{0}$, and the problem

$$
\begin{aligned}
\Delta^{2} \widetilde{\psi} & =0 & & \text { in } S, \\
\widetilde{\psi}=\widetilde{\psi}_{z z}-\widetilde{\psi}_{x x} & =0 & & \text { on } z=0, x \in[-M, M], \\
\widetilde{\psi}_{x}=\widetilde{\psi}_{z} & =0 & & \text { on } z=-1, x \in[-M, 0], \\
\widetilde{\psi}_{z z}-\widetilde{\psi}_{x x} & =0 & & \text { on } z=-1, x \in[0, M], \\
3 \widetilde{\psi}_{x x z}+\widetilde{\psi}_{z z z}+\gamma \widetilde{\psi}_{x} & =-\gamma \psi_{x}^{0} & & \text { on } z=-1, x \in[0, M], \\
\widetilde{\psi}_{z}=\widetilde{\psi}_{x} & =0 & & \text { at } x= \pm M .
\end{aligned}
$$

By introducing $\sum_{n=1}^{\infty} \gamma^{n} \psi^{(n)}$ into $5.8-5.13$ and retaining terms of the same order in $\gamma$ we can write the following problems $P_{j+1}$ :

$$
\begin{array}{rlrl}
\Delta^{2} \psi^{(j+1)} & =0 & & \text { in } S, \\
\psi^{(j+1)}=\psi_{z z}^{(j+1)}-\psi_{x x}^{(j+1)}=0 & & \text { on } z=0, x \in[-M, M], \\
\psi_{x}^{(j+1)}=\psi_{z}^{(j+1)}=0 & & \text { on } z=-1, x \in[-M, 0], \\
\psi_{z z}^{(j+1)}-\psi_{x x}^{(j+1)}=0 & & \text { on } z=-1, x \in[0, M], \\
3 \psi_{x x z}^{(j+1)}+\psi_{z z z}^{(j+1)}=-\psi_{x}^{(j)} & & \text { on } z=-1, x \in[0, M], \\
\psi_{z}^{(j+1)}=\psi_{x}^{(j+1)}=0 & \text { at } x= \pm M,
\end{array}
$$

with $\psi_{x}^{(j)}$ considered as a source. Writing the problems $P_{0}$ and $P_{j+1}$ in terms of the stress tensor as we did in Section 3 and applying Lax-Milgram we can prove existence and uniqueness of solutions and the estimates

$$
\left\|\psi^{(0)}\right\|_{H^{2}(S)} \leqslant C_{0}, \quad\left\|\psi^{(j+1)}\right\|_{H^{2}(S)} \leqslant C_{1}\left\|\psi_{x}^{(j)}(x,-1)\right\|_{L^{2}([0, M])},
$$

where $C_{0}$ depends on the inflow and outflow terms and $C_{1}$ depends only on the domain $S$. By the trace inequality, $\left\|\psi_{x}^{(j)}(x,-1)\right\|_{L^{2}([0, M])}$ is bounded by $\left\|\psi^{(j)}\right\|_{H^{2}(S)}$ and we conclude that

$$
\left\|\psi^{(j+1)}\right\|_{H^{2}(S)} \leqslant C\left\|\psi^{(j)}\right\|_{H^{2}(S)},
$$

where $C$ depends solely on the domain $S$. Hence

$$
\left\|\psi^{(j+1)}\right\|_{H^{2}(S)} \leqslant C^{j+1}\left\|\psi^{(0)}\right\|_{H^{2}(S)} \leqslant C^{j+1} C_{0},
$$

and the series 5.7 is convergent provided $\gamma C<1$, i.e. $\gamma<\gamma_{0} \equiv 1 / C$; this concludes the proof of the theorem. 


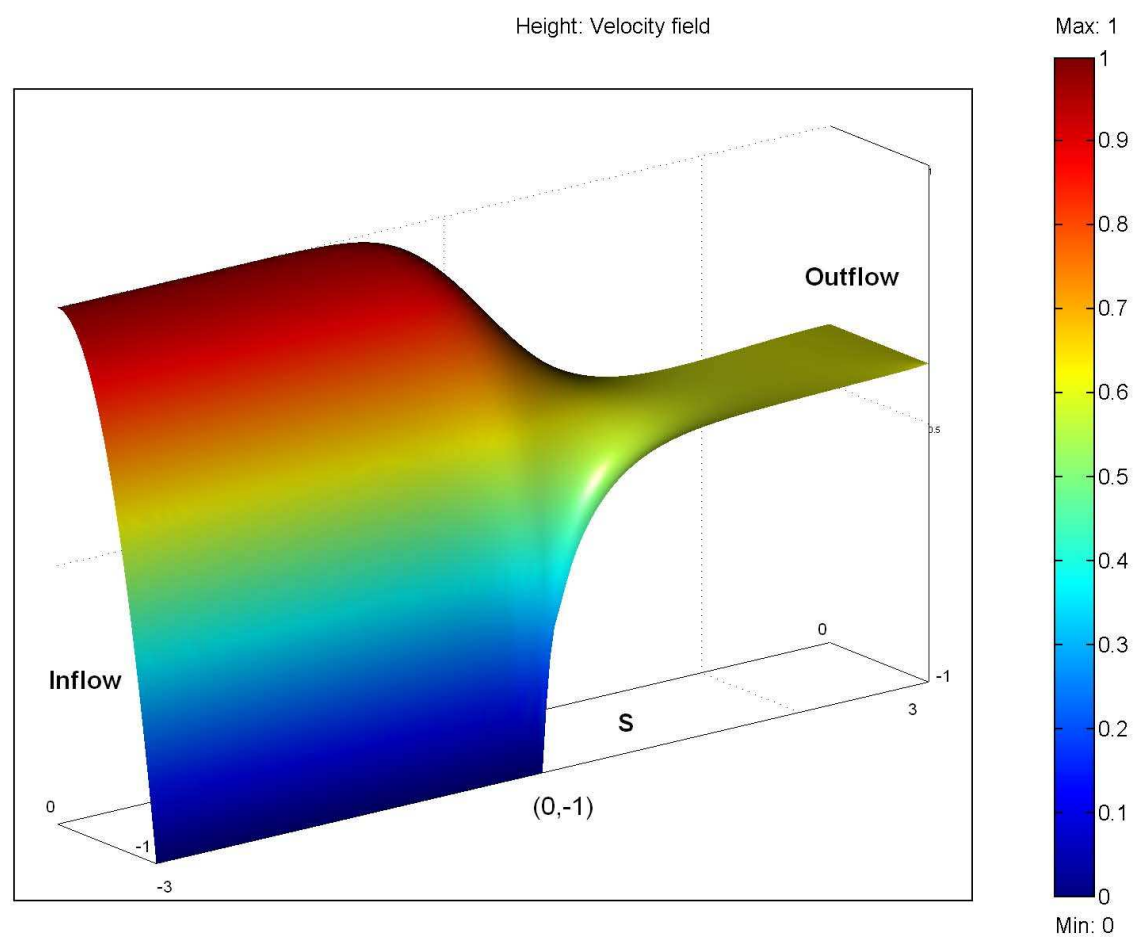

FIG. 3. Modulus of the velocity across the strip. Notice the behavior near $(0,-1)$.

The theorem above and formula 2.13 allow us to represent the free boundary in the form

$$
b(x)=-\frac{1}{U} \psi(x,-1)=\sum_{n=0}^{\infty} \gamma^{n} g_{n}(x)
$$

where

$$
g_{0}(x)=-\frac{1}{U} \psi^{0}(x,-1), \quad g_{n}(x)=-\frac{1}{U} \psi^{(n)}(x,-1) .
$$

By trace embeddings, it follows that $\left\|\psi^{(n)}(x,-1)\right\|_{C^{0}([0, M])} \leqslant C\left\|\psi^{(n)}\right\|_{H^{2}(S)}$ and one can therefore write

$$
b(x)=g_{0}(x)+\gamma g_{1}(x)+O\left(\gamma^{2}\right)
$$

if $\gamma$ is small enough. Formula (5.20) gives a useful representation for the free boundary that can be implemented numerically. We have solved the problem $P_{0}$ in the domain $S=[-3,3] \times[-1,0]$ by using finite elements with inflow and outflow at the lateral boundaries given by

$$
\left(v_{x}^{\text {in }}, v_{z}^{\text {in }}\right)=\left(1-z^{2}, 0\right), \quad\left(v_{x}^{\text {out }}, v_{z}^{\text {out }}\right)=\left(U_{\infty}, 0\right),
$$

where $U_{\infty}$ is chosen such that the total inflow across $x=-3$ is larger than the total outflow across $x=3$, i.e.

$$
U_{\infty}=\int_{-1}^{0}\left(1-z^{2}\right) \mathrm{d} z-\delta=\frac{2}{3}-\delta
$$




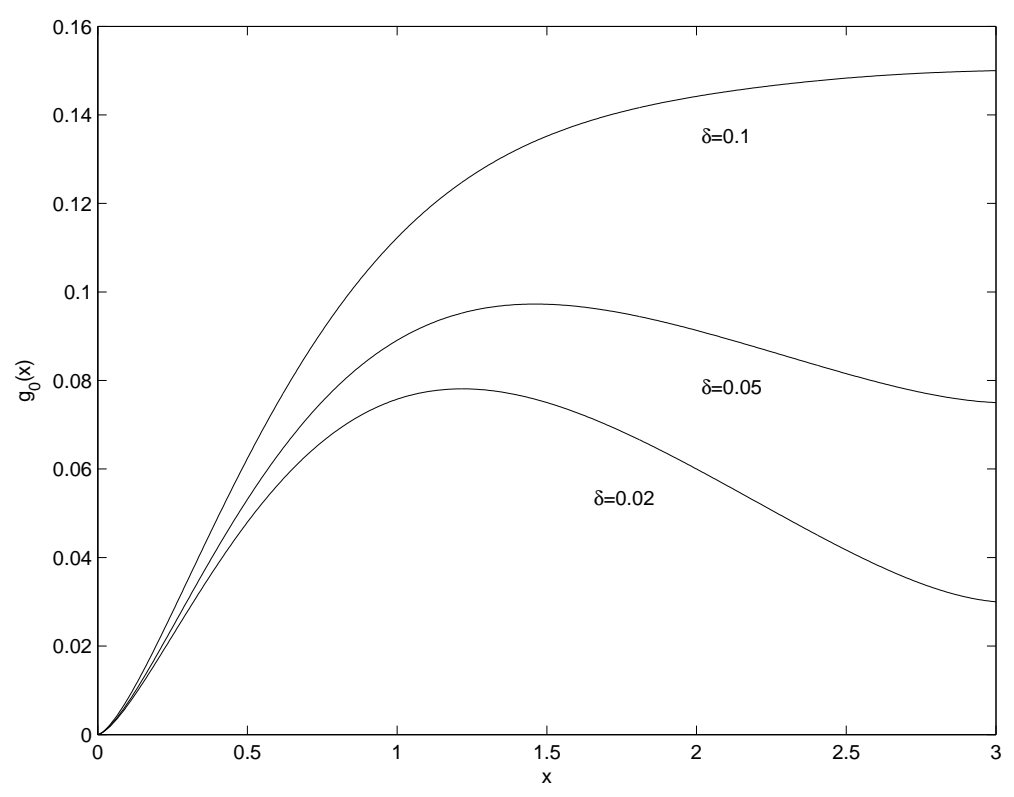

FIG. 4. The free boundary $g_{0}(x)$ for three different values of $\delta$.

In Figure 3 we represent the modulus of the velocity with outflow $U_{\infty}=2 / 3-0.005$. Notice the behavior near $(0,-1)$ suggesting a singularity in the derivative of the velocity. In fact, we proved in the previous sections that $|\vec{v}|=O\left(r^{1 / 2}\right)$. In Figure 4 we represent the first order term $g_{0}(x)$ (see formula (5.20) ) of the representation of the free boundary as a power series in $\gamma$. If $\gamma$ is small, then the free boundary is very similar to the profiles represented in the figure. We have taken $\delta=0.02,0.05,0.1$. Notice the regular behavior near $x=0$. The theory developed in the previous sections predicts $g_{0}(x)=O\left(x^{3 / 2}\right)$ near $x=0$.

\section{The nonlinear problem. Proof of Theorem 1.1}

In this section we present the proof of Theorem 1.1 which states the existence of a unique solution of the nonlinear problem under certain assumptions on the inflow and outflow, and which also describes the asymptotic behavior of the free boundary near the grounding line. We shall split the proof of the theorem into several steps to make the argument easier to follow.

Step 1: Existence and uniqueness of flows for a given geometry. Firstly, we assume that the interface $\Gamma_{2}$ is sufficiently smooth, say $C^{1+\delta}$, and construct a weak solution to the flow problem in a given domain via Lax-Milgram's theorem. We write, as we did for the linearized problem, $\vec{v}=\vec{v}^{0}+\vec{v}^{1}$ where $\vec{v}^{0}$ is a divergence free and regular velocity field satisfying the inflow and outflow boundary conditions as well as the conditions at $z=0$ and the no-slip boundary conditions at $(x,-1), x<0$. Then the problem can be expressed in the form

$$
-\nabla p+\Delta \vec{v}^{1}=\vec{J} \quad \text { in } D
$$




$$
\begin{aligned}
\nabla \cdot \vec{v}^{1} & =0 \quad \text { in } D, \\
\vec{v}^{1} & =0 \quad \text { in } \Gamma_{1}, \\
T\left(\vec{v}^{1}\right) \vec{n} & =-\gamma\left(\psi^{0}+\psi^{1}\right) \vec{n}-T\left(\vec{v}^{0}\right) \vec{n} \quad \text { in } \Gamma_{2}, \\
\vec{v}^{1} \cdot \vec{n} & =0 \quad \text { in } \Gamma_{0}, \\
\vec{t} T\left(\vec{v}^{1}\right) \vec{n} & =0 \quad \text { in } \Gamma_{0}, \\
\vec{v}^{1} & =0 \quad \text { at } x= \pm M,
\end{aligned}
$$

where $T\left(\vec{v}^{0}\right)$ is the stress tensor computed for the velocity field $\vec{v}^{0}$ with $p^{0}=0$. Multiplication by a vector field $\vec{\varphi}=\left(\varphi_{1}, \varphi_{2}\right)$ vanishing at $x= \pm M$ and $\Gamma_{1}$ and such that $\varphi_{2}=0$ at $\Gamma_{0}$, followed by integration by parts, yields the formula

$$
\begin{aligned}
\frac{1}{2} \int_{D}\left(v_{i, j}^{1}+v_{j, i}^{1}\right)\left(\varphi_{i, j}+\right. & \left.\varphi_{j, i}\right) \mathrm{d} x \mathrm{~d} y=-\int_{D} \vec{\varphi} \cdot \vec{J} \mathrm{~d} x \mathrm{~d} y \\
& +\int_{\Gamma_{2}}\left(\left(\vec{t} T\left(\vec{v}^{0}\right) \vec{n}\right) \vec{\varphi} \cdot \vec{t}+\left(\vec{n} T\left(\vec{v}^{0}\right) \vec{n}-\gamma\left(\psi^{0}+\psi^{1}\right) b(x)\right) \vec{\varphi} \cdot \vec{n}\right) \mathrm{d} s .
\end{aligned}
$$

If we introduce in 6.8 a stream function $\omega$ associated to $\vec{\varphi}$ then we can write

$$
\begin{aligned}
\frac{1}{2} \int_{D}\left(v_{i, j}^{1}+v_{j, i}^{1}\right)\left(\varphi_{i, j}+\varphi_{j, i}\right) \mathrm{d} x & \mathrm{~d} y-\gamma \int_{0}^{M} \psi^{1}(x, b(x)) \frac{\mathrm{d}}{\mathrm{d} x} \omega(x, b(x)) \mathrm{d} x \\
= & \int_{\Gamma_{2}}\left(\left(\vec{t} T\left(\vec{v}^{0}\right) \vec{n}\right) \vec{\varphi} \cdot \vec{t}+\left(\vec{n} T\left(\vec{v}^{0}\right) \vec{n}\right) \vec{\varphi} \cdot \vec{n}\right) \mathrm{d} s \\
& +\gamma \int_{\Gamma_{2}} \psi^{0}(x, b(x)) \vec{\varphi} \cdot \vec{n} \mathrm{~d} s-\int_{D} \vec{\varphi} \cdot \vec{J} \mathrm{~d} x \mathrm{~d} y \equiv\langle f, \vec{\varphi}\rangle .
\end{aligned}
$$

This formulation allows direct application of the Lax-Milgram theorem together with Korn's inequality and Sobolev embeddings, yielding

$$
\left\|\vec{v}^{1}\right\|_{H^{1}(D)}^{2} \leqslant C \int_{D}\left(v_{i, j}^{1}+v_{j, i}^{1}\right)^{2} \mathrm{~d} x \mathrm{~d} y
$$

to conclude the existence of a velocity field $\vec{v}^{1} \in\left(H^{1}(D)\right)$ (or equivalently a stream function $\left.\psi^{1} \in H^{2}(D)\right)$ satisfying (6.1)-6.7) and such that

$$
\int_{D}\left(v_{i, j}^{1}+v_{j, i}^{1}\right)^{2} \mathrm{~d} x \mathrm{~d} y \leqslant\left(\left\|T\left(\vec{v}^{0}\right) \vec{n}\right\|_{L^{2}\left(\Gamma_{2}\right)}+\gamma\left\|\psi^{0}\right\|_{L^{2}\left(\Gamma_{2}\right)}\right)\left\|\vec{v}^{1}\right\|_{L^{2}\left(\Gamma_{2}\right)}+\|\vec{J}\|_{L^{2}(D)}\left\|\vec{v}^{1}\right\|_{L^{2}(D)},
$$

and hence, using trace inequalities applied to $\Gamma_{2}$ to estimate $\left\|\vec{v}^{1}\right\|_{L^{2}\left(\Gamma_{2}\right)}$ by $\left\|\vec{v}^{1}\right\|_{H^{1}(D)}$, we conclude that

$$
\left\|\vec{v}^{1}\right\|_{H^{1}(D)} \leqslant C\left(\left\|T\left(\vec{v}^{0}\right) \vec{n}\right\|_{L^{2}\left(\Gamma_{2}\right)}+\gamma\left\|\psi^{0}\right\|_{L^{2}\left(\Gamma_{2}\right)}+\|\vec{J}\|_{L^{2}(D)}\right) \leqslant C\left\|\psi^{0}\right\|_{H^{3}(D)} .
$$

Both the Lax-Milgram theorem and Korn's inequality can be applied provided the curve $\Gamma_{2}$ is continuously differentiable (see for instance [4]).

Step 2: Weak formulation of the nonlinear problem. The free boundary problem can be restated as the problem of finding the curve $z=b(x)$ that describes $\Gamma_{2}$ and satisfies

$$
b_{x}=-\frac{1}{U}\left(b_{x}\left(v_{x}^{0}+v_{x}^{1}\right)-\left(v_{z}^{0}+v_{z}^{1}\right)\right) .
$$


The velocity field $\vec{v}^{1}$ such that 6.9 ) and 6.10$)$ hold is found simultaneously. Our strategy to solve (6.9), (6.10) will be to look for a fixed point "near" (in some norm to be defined) the solution to the linearized problem analyzed in the previous sections. This implies that we have to relate the problem defined in the domain $D$ to problems defined in a strip. An application of a diffeomorphism from $D$ to $S=[-M, M] \times[-1,0]$ so that $x^{\prime}=x, z^{\prime}=z-\chi(x, z) b(x)$ leads to a change in the derivatives

$$
\partial_{x}=\partial_{x^{\prime}}+(\chi b)_{x} \partial_{z^{\prime}}, \quad \partial_{z}=(1-\chi z) \partial_{z^{\prime}} .
$$

On the other hand, the line integrals over $\Gamma_{2}$ can be written as

$$
\int_{\Gamma_{2}}(\cdot) \mathrm{d} s=\int_{x}(\cdot)\left(1+b_{x}^{2}\right)^{1 / 2} \mathrm{~d} x=\int_{x}(\cdot) \mathrm{d} x+\int_{x}(\cdot)\left[\left(1+b_{x}^{2}\right)^{1 / 2}-1\right] \mathrm{d} x .
$$

Hence we end up with a reformulation of 6.9 as

$$
\begin{aligned}
a\left(\vec{v}^{1}, \vec{\varphi}\right) \equiv & \frac{1}{2} \int_{S}\left(v_{i, j^{\prime}}^{1}+v_{j, i^{\prime}}^{1}\right)\left(\varphi_{i, j^{\prime}}+\varphi_{j, i^{\prime}}\right) \mathrm{d} x^{\prime} \mathrm{d} y^{\prime} \\
& -\gamma \int_{0}^{M} \psi^{1}\left(x^{\prime},-1\right) \frac{\mathrm{d}}{\mathrm{d} x^{\prime}} \omega\left(x^{\prime},-1\right) \mathrm{d} x^{\prime}=\left\langle f^{\prime}, \vec{\varphi}\right\rangle+\left\langle G\left(\vec{v}^{1}, b\right), \vec{\varphi}\right\rangle,
\end{aligned}
$$

where

$$
\begin{aligned}
\left\langle f^{\prime}, \vec{\varphi}\right\rangle= & \int_{0}^{M}\left(\left(\vec{t} T\left(\vec{v}^{0}\right) \vec{n}\right) \varphi_{x^{\prime}}+\left(\vec{n} T\left(\vec{v}^{0}\right) \vec{n}\right) \varphi_{z^{\prime}}\right) \mathrm{d} x \\
& +\gamma \int_{0}^{M} \psi^{0}(x, b(x)) \varphi_{z^{\prime}} \mathrm{d} x \leqslant C\left\|\psi^{0}\right\|_{H^{3}(S)}\|\vec{\varphi}\|_{H^{1}(S)},
\end{aligned}
$$

and $G\left(\vec{v}^{1}, b\right)$ contains the terms $(\chi b)_{x} \partial_{z^{\prime}} v_{i}$ and $\chi_{z} b \partial_{z^{\prime}} v_{i}$, functions of $\psi^{0}$ and their derivatives multiplied by $O\left(b_{x}\right)$ factors together with the term $\left(v_{i, j^{\prime}}^{1}+v_{j, i^{\prime}}^{1}\right)(1-J)$ with $J$ being the determinant of the Jacobian matrix of the transformation, which involves derivatives of $b$ up to the first order. Notice that, in any case, one can estimate

$$
\left\langle G\left(\vec{v}^{1}, b\right), \vec{\varphi}\right\rangle \leqslant C\|b\|_{W^{1, \infty}}\left(\left\|\psi^{0}\right\|_{H^{3}(S)}+\left\|\vec{v}^{1}\right\|_{H^{1}(S)}\right)\|\vec{\varphi}\|_{H^{1}(S)},
$$

where $\|b\|_{W^{1, \infty}} \equiv \sup _{x}|b|+\sup _{x}\left|b_{x}\right|$ is supposed to be small enough. The equation $a\left(\vec{v}^{1}, \vec{\varphi}\right)=$ $\left\langle f^{\prime}, \vec{\varphi}\right\rangle$ is the linearized problem solved in the previous sections, yielding a solution $\vec{v}^{1}$ such that $\left\|\vec{v}^{1}\right\|_{H^{1}(S)} \leqslant C\left\|\psi^{0}\right\|_{H^{3}(S)}$. On the other hand, equation 6.10 can be written in the following form:

$$
b_{x}=\frac{1}{U}\left(v_{z}^{0}+v_{z}^{1}\right)-\frac{1}{U} b_{x}\left(v_{x}^{0}+v_{x}^{1}\right) \equiv \frac{1}{U}\left(v_{z}^{0}+v_{z}^{1}\right)+F\left[\vec{v}^{0}+\vec{v}^{1}, b_{x}\right] .
$$

The equation $b_{x}=(1 / U)\left(v_{z}^{0}+v_{z}^{1}\right)$ is the linearized version of equation 6.10 and is the one considered in the previous sections to obtain the profile $b(x)$.

Step 3: Setting and resolution of the fixed point problem. In order to solve 6.11, (6.12) let us consider the problem

$$
\begin{aligned}
a\left(\vec{v}^{1}, \vec{\varphi}\right) & =\left\langle f^{\prime}, \vec{\varphi}\right\rangle+\langle G(\vec{w}, \bar{b}), \vec{\varphi}\rangle, \\
b_{x} & =\frac{1}{U}\left(v_{z}^{0}+v_{z}^{1}\right)+F\left[\overrightarrow{v^{0}}+\vec{w}, \bar{b}_{x}\right],
\end{aligned}
$$


which represents a nonlinear mapping $T$ from $(\vec{b}, \vec{w})$ to the solution $\left(b, \vec{v}^{1}\right)$. The solution we wish to find is a fixed point of the mapping $T$. If $\vec{w} \in H^{1}(S)$ and $b \in W^{1, \infty}$ then by Lax-Milgram again, 6.13 has a unique solution satisfying

$$
\left\|\vec{v}^{1}\right\|_{H^{1}(S)} \leqslant C\left\|\psi^{0}\right\|_{H^{3}(S)}+C\|\bar{b}\|_{W^{1, \infty}}\left(\left\|\psi^{0}\right\|_{H^{3}(S)}+\|\vec{w}\|_{H^{1}(S)}\right) .
$$

In fact, we will need higher regularity of $\vec{v}^{1}$. This can be achieved by introducing suitable partitions of unity, local chart diffeomorphisms and classical elliptic estimates (cf. for instance [2]). Since the process is quite straightforward, but lengthy, we omit the details and simply write the estimate:

$$
\left\|\vec{v}^{1}\right\|_{H^{1+\delta}(S)} \leqslant C\left\|\psi^{0}\right\|_{H^{3}(S)}+C\|\bar{b}\|_{C^{1+\delta}}\left(\left\|\psi^{0}\right\|_{H^{3}(S)}+\|\vec{w}\|_{H^{1+\delta}(S)}\right)
$$

By trace inequalities, we can estimate the $H^{1 / 2+\delta}$ norm of $\vec{v}^{1}$ on the line given by $\{(x,-1): x \in$ $[0, M]\}$ in terms of $\left\|\vec{v}^{1}\right\|_{H^{1+\delta}(S)}$, and by the Sobolev embedding of $C^{\delta}$ in $H^{1 / 2+\delta}$ we conclude that

$$
\left\|\vec{v}^{1}(x,-1)\right\|_{C^{\delta}} \leqslant C\left\|\psi^{0}\right\|_{H^{3}(S)}+C\|\bar{b}\|_{C^{1+\delta}}\left(\left\|\psi^{0}\right\|_{H^{3}(S)}+\|\vec{w}\|_{H^{1+\delta}(S)}\right) .
$$

It is a direct calculation to deduce from 6.14 that

$$
\begin{aligned}
\|b\|_{C^{1+\delta}} & \leqslant \frac{1}{U}\left(\left\|v_{z}^{0}\right\|_{C^{\delta}}+\left\|v_{z}^{1}\right\|_{C^{\delta}}\right)+C\|\bar{b}\|_{C^{1+\delta}}\left(\left\|\overrightarrow{v^{0}}\right\|_{C^{\delta}}+\|\vec{w}\|_{C^{\delta}}\right) \\
& \leqslant \frac{1}{U}\left(\left\|\psi^{0}\right\|_{H^{3}(S)}+\left\|\vec{v}^{1}\right\|_{H^{1+\delta}(S)}\right)+C\|\bar{b}\|_{C^{1+\delta}}\left(\left\|\psi^{0}\right\|_{H^{3}(S)}+\|\vec{w}\|_{H^{1+\delta}(S)}\right) .
\end{aligned}
$$

From 6.15) and 6.16 we conclude

$$
\begin{aligned}
\|b\|_{C^{1+\delta}}+\left\|\vec{v}^{1}\right\|_{H^{1+\delta}(S)} \leqslant & C\left\|\psi^{0}\right\|_{H^{2}(S)}+C\left\|\psi^{0}\right\|_{H^{2}(S)}\left(\|\bar{b}\|_{C^{1+\delta}}+\|\vec{w}\|_{H^{1+\delta}(S)}\right) \\
& +C\|\bar{b}\|_{C^{1+\delta}}\|\vec{w}\|_{H^{1+\delta}(S)},
\end{aligned}
$$

so that $T$ maps the ball of radius $\varepsilon$ in $C^{1+\delta}(0, M) \times H^{2}(S)$ to a ball of radius $R=C\left\|\psi^{0}\right\|_{H^{3}(S)}(1+\varepsilon)$ $+C \varepsilon^{2}$ which is smaller than $\varepsilon$ if both $\varepsilon$ and $\left\|\psi^{0}\right\|_{H^{3}(S)}$ are small enough. Since the problems 6.13, 6.14) are linear, it is simple to verify that the mapping $T$ is also a contraction if $\varepsilon$ is small enough. Hence, by the Banach fixed point theorem there exists a unique solution to the nonlinear free boundary problem.

Step 4: Asymptotic behavior of the free boundary near the grounding line. Notice that by (6.14),

$$
\begin{aligned}
b(x) & =\int_{0}^{x}\left(\frac{1}{U}\left(v_{z}^{0}+v_{z}^{1}\right)+F\left[\vec{v}^{0}+\vec{v}^{1}, b_{x}\right]\right) \mathrm{d} x \\
& \leqslant C x^{1+\delta} \sup \left(\frac{1}{x^{\delta}}\left|\frac{1}{U}\left(v_{z}^{0}+v_{z}^{1}\right)+F\left[{\overrightarrow{v^{0}}}^{1} \vec{v}^{1}, b_{x}\right]\right|\right) \leqslant C x^{1+\delta},
\end{aligned}
$$

so that the interface is flat near $(0,-1)$. This allows us to apply a local cut-off function in the neighborhood of the grounding line and a diffeomorphism to a half-plane. Then the analysis of Section 4.1 is valid and we arrive at the following asymptotic behavior for $b(x)$ :

$$
b(x)=C x^{3 / 2}+o\left(x^{3 / 2}\right) \quad \text { near } x=0 .
$$

This concludes the proof of the theorem.

REMARK 6.1 Since the free boundary is $C^{1+\delta}$, it can be shown, just as in the linear case, that the weak solutions are in fact strong (i.e., the stream function is $C^{4}$ ) inside $D$ and up to the boundaries outside $(0,-1)$. 


\section{Appendix: A Hardy type inequality}

This appendix is devoted to the proof of the following lemma:

LEMMA 7.1 Let $g$ be such that

$$
\|g\|_{H_{\alpha}^{1}}^{2} \equiv \begin{cases}\int_{0}^{\infty} x^{2 \alpha-2}|(g(x)-g(0))|^{2} \mathrm{~d} x+\int_{0}^{\infty} x^{2 \alpha}\left|g_{x}(x)\right|^{2} \mathrm{~d} x<\infty & \text { if } \alpha<1 / 2, \\ \int_{0}^{\infty} x^{2 \alpha-2}|g(x)|^{2} \mathrm{~d} x+\int_{0}^{\infty} x^{2 \alpha}\left|g_{x}(x)\right|^{2} \mathrm{~d} x<\infty & \text { if } \alpha>1 / 2 .\end{cases}
$$

Then the following estimates hold:

$$
\begin{aligned}
\sup _{x \in \mathbb{R}^{+}} \frac{|g(x)-g(0)|}{x^{1 / 2-\alpha}} \leqslant C \int_{0}^{\infty} x^{2 \alpha}\left|g_{x}(x)\right|^{2} \mathrm{~d} x \quad & \text { if } \alpha<1 / 2, \\
\sup _{x \in \mathbb{R}^{+}} \frac{|g(x)|}{x^{1 / 2-\alpha}} \leqslant C \int_{0}^{\infty} x^{2 \alpha}\left|g_{x}(x)\right|^{2} \mathrm{~d} x \quad & \text { if } \alpha>1 / 2 .
\end{aligned}
$$

Proof. We prove the inequalities for functions $g$ defined in $\mathbb{R}^{+}$which are $C^{1}$ and bounded and decay exponentially at infinity. Such functions are dense in $H_{\alpha}^{1}$ so that the inequalities extend automatically to any function in $H_{\alpha}^{1}$. In the case $\alpha<1 / 2$ we can consider $f(x)=g(x)-g(0)$ so that $f(0)=0$ and

$$
\int_{0}^{\infty} x^{2 \alpha}\left|f_{x}(x)\right|^{2} \mathrm{~d} x=\int_{0}^{\infty} x^{2 \alpha}\left|g_{x}(x)\right|^{2} \mathrm{~d} x<\infty
$$

By taking the Mellin transform

$$
\tilde{f}_{x}^{\alpha}(\lambda)=\int_{0}^{\infty} f_{x}(u) u^{i \lambda+\alpha-1 / 2} \mathrm{~d} u,
$$

where $\lambda \in \mathbb{R}$, and using Plancherel's identity

$$
\int_{0}^{\infty} x^{2 \alpha}\left|f_{x}(x)\right|^{2} \mathrm{~d} x=\frac{1}{2 \pi} \int_{-\infty}^{\infty}\left|\tilde{f_{x}^{\alpha}}(\lambda)\right|^{2} \mathrm{~d} \lambda
$$

together with the following rule (resulting after integration by parts and using $f(0)=0$ ) relating the Mellin transform of $f_{x}$ with the transform of $f$ :

$$
\tilde{f}_{x}^{\alpha}=-(i \lambda-1 / 2+\alpha) \tilde{f}^{\alpha-1}
$$

we deduce that

$$
\int_{0}^{\infty} x^{2 \alpha}\left|f_{x}(x)\right|^{2} \mathrm{~d} x=\frac{1}{2 \pi} \int_{-\infty}^{\infty}\left|\tilde{f}_{x}^{\alpha}(\lambda)\right|^{2} \mathrm{~d} \lambda=\frac{1}{2 \pi} \int_{-\infty}^{\infty}\left((1 / 2-\alpha)^{2}+\lambda^{2}\right)\left|\tilde{f}^{\alpha-1}(\lambda)\right|^{2} \mathrm{~d} \lambda .
$$

Notice next the following relation of Mellin transform and Fourier transform following from the change of variables $u=e^{t}$ :

$$
\tilde{f}^{\alpha}(\lambda)=\int_{0}^{\infty} f(u) u^{i \lambda+\alpha-1 / 2} \mathrm{~d} u=\int_{-\infty}^{\infty} f\left(e^{t}\right) e^{(\alpha+1 / 2) t} e^{i \lambda t} \mathrm{~d} t=F\left(f\left(e^{t}\right) e^{(\alpha+1 / 2) t}\right),
$$


so that

$$
\begin{aligned}
\int_{0}^{\infty} x^{2 \alpha}\left|f_{x}(x)\right|^{2} \mathrm{~d} x & =\frac{1}{2 \pi} \int_{-\infty}^{\infty}\left((1 / 2-\alpha)^{2}+\lambda^{2}\right)\left|F\left(f\left(e^{t}\right) e^{(\alpha-1 / 2) t}\right)\right|^{2} \mathrm{~d} \lambda \\
& \geqslant C_{\alpha} \sup _{t \in \mathbb{R}}\left|f\left(e^{t}\right) e^{(\alpha-1 / 2) t}\right|^{2} \quad(\alpha \neq 1 / 2),
\end{aligned}
$$

where we have used, in the last inequality, the Sobolev embedding $H^{1}(\mathbb{R}) \subset L^{\infty}(\mathbb{R})$. Therefore

$$
\sup _{x \in \mathbb{R}^{+}} \frac{|g(x)-g(0)|^{2}}{x^{1-2 \alpha}}=\sup _{x \in \mathbb{R}^{+}} \frac{|f(x)|^{2}}{x^{1-2 \alpha}} \leqslant C \int_{0}^{\infty} x^{2 \alpha}\left|f_{x}(x)\right|^{2} \mathrm{~d} x=C \int_{0}^{\infty} x^{2 \alpha}\left|g_{x}(x)\right|^{2} \mathrm{~d} x,
$$

proving $(7.1)$. If $\alpha>1 / 2$, then we apply Mellin transform directly to $g_{x}$ :

$$
\tilde{g_{x}^{\alpha}}(\lambda)=\int_{0}^{\infty} g_{x}(u) u^{i \lambda+\alpha-1 / 2} \mathrm{~d} u
$$

and the rule for the derivative

$$
\tilde{g}_{x}^{\alpha}(\lambda)=-(i \lambda-1 / 2+\alpha) \tilde{g}^{\alpha-1}(\lambda)
$$

which can be deduced by integrating by parts. Then the same reasoning as above yields 7.2 .

\section{Acknowledgements}

We thank A. C. Fowler for proposing this problem and sharing some of his ideas with us, and J. L. Cubas for his help in producing the figures.

\section{REFERENCES}

1. Agmon, S., Douglis, A., \& Nirenberg, L. Estimates near the boundary for solutions of elliptic partial differential equations satisfying general boundary conditions, I. Comm. Pure Appl. Math. 12 (1959), 623-727. Zbl 0093.10401 MR 0125307

2. BreZIS, H. Analyse fonctionnelle: Théorie et applications. Masson, Paris (1983). Zbl 0511.46001 MR 0697382

3. Davis, S. H., Kistler, S., \& Brown, R. (eds.). Free Boundaries in Viscous Flows. IMA Vol. Math. Appl. 61, Springer (1994). Zbl 0790.00010 MR 1301782

4. Duvaut, G., \& Lions, J.-L. Inequalities in Mechanics and Physics. Springer (1976). Zbl 0331.35002 MR 0521262

5. Fontelos, M. A., \& Velázquez, J. J. L. A free boundary problem for the Stokes system with contact lines. Comm. Partial Differential Equations 23 (1998), 1209-1303. Zbl 0913.35156 MR 1642615

6. Fowler, A. C. Waves on glaciers. J. Fluid Mech. 120 (1982), 283-321. Zbl 0552.76007 MR 0669435

7. Fowler, A. C. Sliding with cavity formation. J. Glaciology 33 (1987), 255-267.

8. Fowler, A. C., \& LARSON, D. A. On the flow of polythermal glaciers, I: Model and preliminary analysis. Proc. R. Soc. London Ser. A 363 (1978), 217-242. MR 0502581

9. Friedman, A., \& Velázquez, J. J. L. The analysis of coating flows near the contact line. J. Differential Equations 119 (1995), 137-208. Zbl 0851.35106 MR 1334490

10. Friedman, A., \& VelázQuez, J. J. L. The analysis of coating flows in a strip. J. Differential Equations 121 (1995), 134-282. Zbl 0836.76022 MR 1348539 
11. Hindmarsh, R. C. A. Stability of ice rises and uncoupled marine ice sheet. Ann. Glaciology 23 (1996), $105-115$.

12. Hindmarsh, R. C. A., \& Le Meur, E. L. Dynamical processes involved in the retreat of marine ice sheet. J. Glaciology 47 (2001), 271-282.

13. Kondrat'Ev, V. A. Boundary value problems for elliptic equations in domains with conical or angular points. Trudy Moskov. Mat. Obshch. 16 (1967), 209-292 (in Russian); English transl.: Trans. Moscow Math. Soc. 16 (1967), 227-313. Zbl 0194.13405 MR 0226187

14. Kozlov, V. A., Maz'ya, V. G., \& Rossmann, J. Elliptic Boundary Problems in Domains with Point Singularities. Math. Surveys Monogr. 52, Amer. Math. Soc., Providence, RI (1997). Zbl 0947.35004 MR 1469972

15. Kristel, S. F., \& SCRiven, L. E. Chapter 8 in: Coating Flows in Computational Analysis of Polymer Processing, J. R. A. Pearson and S. M. Richardson (eds.), Appl. Sci. Publ., London (1983), 248-299.

16. Kristel, S. F., \& SCRiven, L. E. Coating flow theory by finite element and asymptotic analysis of the Navier-Stokes system. Internat. J. Numer. Methods Fluids 4 (1984), 207-229.

17. Maz'Ya, V. G., Kozlov, V. A., \& Rossmann, J. Spectral Problems Associated with Corner Singularities of Solutions to Elliptic Equations. Math. Surveys Monogr. 85, Amer. Math. Soc. (2001). Zbl 0965.35003 MR 1788991

18. Maz’ya, V. G., Morozov, N. F., PlamenevskiĬ, B. A., \& Stupyalis, L. Elliptic Boundary Value Problems. Amer. Math. Soc. Transl. 123 (1984).

19. NGan, C. C., \& Dussan, E. B. On the dynamics of liquid spreading on solid surfaces. J. Fluid Mech. 209 (1989), 191-226.

20. Plamenevs KIĬ, B. A. Elliptic Boundary Value Problems in Domains with Piecewise Smooth Boundary. Encyclopaedia Math. Sci. 79, Springer, Berlin (1997). Zbl 0880.35003 MR 1481217

21. Puknnachev, V. V. Von Mises variables in problems with free boundary for the Navier-Stokes equations. Dokl. Akad. Nauk SSSR 210 (1973), 298-301 (in Russian). Zbl 0301.76015 MR 0329423

22. Puknnachev, V. V., \& Solonnikov, V. A. On the problem of dynamic contact angle. J. Appl. Math. Mech. 46 (1982), 771-779. Zbl 0532.76026 MR 0726121

23. RAVIART, P. A., \& ThOMAS, J. M. Introduction à l'analyse numérique des équations aux dérivées partielles. Collection Mathématiques Appliquées pour la Maitrise, Masson, Paris (1983). Z Zbl 0561.65069 MR 0773854

24. Sherperd, A., Wingham, D. J., Mansley, J. A. D., \& Corr, H. F. J. Inland thinning of Pine Island Glacier, West Antarctica. Science 291-5505 (2001), 862-864.

25. Solonnikov, V. A. Solvability of a problem on the plane motion of heavy viscous incompressible capillary liquid partially filling a container. Math. USSR-Izv. 14 (1980), 193-221. Zbl 0429.76020 MR 0525947

26. Solonnikov, V. A. On some free boundary problems for the Navier-Stokes equations with moving contact points and lines. Math. Ann. 302 (1995), 743-772. Zbl 0926.35116 MR 1343648

27. Vieli, A., \& PAYNe, A. J. Assessing the ability of numerical ice sheet models to simulate grounding line migration. J. Geophys. Res.-Earth Surface 110 (2005), Art. No F01003.

28. Wilchinsky, A. V., \& Chugunov, V. A. Modelling of a marine glacier and ice sheet-ice shelf transition zone based on asymptotic analysis. Ann. Glaciology 23 (1996), 59-67.

29. Wilchinsky, A. V., \& Chugunov, V. A. Ice stream-ice shelf transition: theoretical analysis of twodimensional flow. Ann. Glaciology 30 (2000), 153-162. 